\section{Beta thalassemia minor is a beneficial determinant of red blood cell storage lesion}

\author{
Vassilis L. Tzounakas, ${ }^{1 \star}$ Alkmini T. Anastasiadi, ${ }^{1 *}$ Davide Stefanoni, ${ }^{2}$ Francesca \\ Cendali, ${ }^{2}$ Lorenzo Bertolone, ${ }^{2}$ Fabia Gamboni, ${ }^{2}$ Monika Dzieciatkowska, ${ }^{2}$ \\ Pantelis Rousakis, ${ }^{3}$ Athina Vergaki, ${ }^{4}$ Vassilis Soulakis, ${ }^{4}$ Ourania E. Tsitsilonis, ${ }^{3}$ \\ Konstantinos Stamoulis, ${ }^{5}$ Issidora S. Papassideri, ${ }^{1}$ Anastasios G. Kriebardis, ${ }^{6}$ \\ Angelo D'Alessandro ${ }^{2}$ and Marianna H. Antonelou ${ }^{1}$
}

Haematologica 2022

Volume 107(1):112-125

${ }^{1}$ Department of Biology, Section of Cell Biology and Biophysics, School of Science, National and Kapodistrian University of Athens (NKUA), Athens, Greece; ${ }^{2}$ Department of Biochemistry and Molecular Genetics, University of Colorado, School of MedicineAnschutz Medical Campus, Aurora, CO, USA; ${ }^{3}$ Department of Biology, Section of Animal and Human Physiology, School of Science (NKUA), Athens, Greece; ${ }^{4}$ Regional Blood Transfusion Center, "Agios Panteleimon" General Hospital of Nikea, Piraeus, Greece; ${ }^{5}$ Hellenic National Blood Transfusion Center, Acharnes, Athens, Greece and ${ }^{6}$ Department of Biomedical Science, School of Health \& Caring Science, University of West Attica (UniWA), Egaleo, Greece.

*VLT and ATA contributed equally as co-first authors.

\section{ABSTRACT}

B lood donor genetics and lifestyle affect the quality of red blood cell (RBC) storage. Heterozygotes for beta thalassemia $\left(\beta T_{h a l}{ }^{+}\right)$ constitute a non-negligible proportion of blood donors in the Mediterranean and other geographical areas. The unique hematological profile of $\beta \mathrm{Thal}^{+}$could affect the capacity of enduring storage stress, however, the storability of $\beta T_{h a l^{+}} \mathrm{RBC}$ is largely unknown. In this study, RBC from $18 \beta \mathrm{Thal}^{+}$donors were stored in the cold and profiled for primary (hemolysis) and secondary (phosphatidylserine exposure, potassium leakage, oxidative stress) quality measures, and metabolomics, versus sex- and age-matched controls. The $\beta$ Thal ${ }^{+}$units exhibited better levels of storage hemolysis and susceptibility to lysis following osmotic, oxidative and mechanical insults. Moreover, $\beta \mathrm{Thal}^{+}$ $\mathrm{RBC}$ had a lower percentage of surface removal signaling, reactive oxygen species and oxidative defects to membrane components at late stages of storage. Lower potassium accumulation and higher uratedependent antioxidant capacity were noted in the $\beta \mathrm{Thal}^{+}$supernatant. Full metabolomics analyses revealed alterations in purine and arginine pathways at baseline, along with activation of the pentose phosphate pathway and glycolysis upstream to pyruvate kinase in $\beta T_{h a l^{+}} \mathrm{RBC}$. Upon storage, substantial changes were observed in arginine, purine and vitamin B6 metabolism, as well as in the hexosamine pathway. A high degree of glutamate generation in $\beta T_{h a l^{+}} \mathrm{RBC}$ was accompanied by low levels of purine oxidation products (IMP, hypoxanthine, allantoin). The $\beta$ Thal mutations impact the metabolism and the susceptibility to hemolysis of stored RBC, suggesting good post-transfusion recovery. However, hemoglobin increment and other clinical outcomes of $\beta$ Thal $^{+} \mathrm{RBC}$ transfusion deserve elucidation by future studies.

\section{Introduction}

Inter-donor heterogeneity significantly impacts the two "gold standards" of red blood cell (RBC) storage quality, namely end of storage hemolysis and in vivo 24hour post-transfusion recovery. ${ }^{1}$ Donor age, sex, ethnicity ${ }^{2}$ and lifestyle (smoking, drinking, caffeine consumption ${ }^{3}$ ) all impact stored RBC energy and redox metabolism, and thereby RBC capacity to cope with oxidant and other insults. These factors ultimately affect transfusion efficacy, as gleaned by outcomes like hemoglobin $(\mathrm{Hb})$ increments upon transfusion. ${ }^{4}$ Genetic factors impacting RBC redox status and antioxidant capacity have been linked to alteration of the metabolic age 
of stored RBC, ${ }^{5}$ such as deficiency in the activity of glucose 6-phosphate dehydrogenase (G6PD) resulting both in increased oxidant stress ${ }^{6}$ and lower recovery in healthy blood donors. ${ }^{7}$ Like G6PD deficiency, genetic polymorphisms associated with non-canonical hemoglobin traits are enriched in certain donor populations. Carrier state for beta thalassemia $\left(\beta \mathrm{Thal}^{+}\right)$is characterized by mild effects on globin synthesis and RBC survival, and as such, many $\beta$ Thal $^{+}$subjects are eligible blood donors. ${ }^{8}$ Despite their unique hematological profile, that could affect the capacity of enduring storage stress in either a negative or a positive way, little is known about the storability and recovery of $\beta$ Thal ${ }^{+} \mathrm{RBC}$.

Apart from $\mathrm{RBC}$ indices and minor $\mathrm{Hb}$ variants, ${ }^{9} \beta \mathrm{Thal}^{+}$ $\mathrm{RBC}$ may exhibit differences in membrane structure, ${ }^{10}$ deformability and ion exchange, ${ }^{11}$ among others. Free heme and iron reactions bring about mild but sustained oxidative stress that when combined with decreased plasma antioxidant capacity, ${ }^{12}$ may lead to oxidative defects in skeletal proteins ${ }^{13}$ and membrane lipids. Augmented protein phosphorylation ${ }^{14}$ and proteolytic cleavage of band $3^{15}$ have been also observed as a probable result of caspase- 3 activation. Since the N-terminus of band 3 can regulate metabolic fluxes through glycolysis by means of inhibitory binding to glycolytic enzymes, the aforementioned alterations could trigger excessive consumption of glucose through the glycolytic pathway at the expense of NADPH production by the pentose phosphate pathway (PPP), ${ }^{11}$ with a consequent deficit in the capacity to fuel several antioxidant systems that rely on this cofactor. Many of these $\beta \mathrm{Thal}^{+} \mathrm{RBC}$ distortions are typical storage lesion aspects and some of them have already been linked to poor recovery or adverse transfusion effects.

On the other hand, the geometry ${ }^{16}$ and membrane cation permeability of $\beta \mathrm{Thal}^{+} \mathrm{RBC}$ render them osmotically resistant, a probably advantageous feature for $\mathrm{RBC}$ at storage conditions. ${ }^{17}$ Of note, baseline adult hemoglobin $\mathrm{A}_{2}\left(\mathrm{HbA}_{2}\right)$ and fetal hemoglobin $(\mathrm{HbF})$ levels in the general donor population have been found to be positively associated with resistance of stored $\mathrm{RBC}$ to stress hemolysis. ${ }^{18}$ Moreover, $\beta$ Thal ${ }^{+}$RBC exhibit low aggregability, ${ }_{19}$ probably rendering them less susceptible to the storage-induced tendency to cell aggregation. Improved metabolic ${ }^{20}$ and total cardiovascular risk profiles, ${ }^{21}$ along with survival following malaria infection ${ }^{22}$ have been also reported thalassemia traits. The aim of the present study was to clarify whether the homeostasis of these unique $\mathrm{RBC}$ acts positively or negatively towards the challenges of blood banking.

\section{Methods}

\section{Biological samples and blood unit preparation}

Venous blood from $n=204$ regular blood donors was collected into EDTA, citrate and serum vacutainer tubes (in vivo study). Thirty-eight donors (18 $\beta \mathrm{Thal}^{+}$and 20 controls) were selected to evaluate RBC storability in citrate-phosphate-dextrose (CPD)/ saline-adenine-glucose-mannitol (SAGM) for 42 days at $4^{\circ} \mathrm{C}$. The two donor groups exhibited typical hematological differences between them but minimal baseline variation in sex, age, donation frequency and other demographics (Online Supplementary Table S1). $\beta \mathrm{Thal}^{+}$trait was confirmed by $\mathrm{Hb}$ electrophoresis and molecular identification of mutations. The study was approved by the Ethics Committee of the Department of
Biology, School of Science, NKUA. Investigations were carried out upon donor consent, in accordance with the principles of the Declaration of Helsinki.

\section{Physiological parameters}

Free $\mathrm{Hb}$ levels were measured in plasma/supernatant through spectrophotometry, ${ }^{23}$ followed by the Allen correction. In order to examine the osmotically induced hemolysis, RBC were exposed to solutions of increasing saline $(\mathrm{NaCl})$ concentration and the mean corpuscular fragility (MCF, concentration of $\mathrm{NaCl}$ at $50 \%$ hemolysis) was calculated. The mechanical fragility index (MFI) was determined by measuring the amount of $\mathrm{Hb}$ released in the supernatant of $\mathrm{RBC}$ rocked with stainless steel beads for 1 hour $(\mathrm{h})$. Oxidative hemolysis levels were evaluated following treatment of RBC with $17 \mathrm{mM}$ phenylhydrazine (PHZ) for $1 \mathrm{~h}$ at $37^{\circ} \mathrm{C}$. Reactive oxygen species (ROS) and calcium accumulation were measured by fluorometry; the extracellular antioxidant activity and lipid peroxidation were determined spectrophotometrically; phosphatidylserine (PS) exposure and RBC membrane protein carbonylation were estimated by multicolor flow cytometry and western blotting, respectively (see the Online Supplementary Methods for details).

\section{Omics analyses}

Preliminary proteomics analyses were performed in stored samples of RBC membranes through a filter-aided sample preparation digestion prior to analysis via nano-ultra performance liquid chromatography - tandem mass spectrometer (nanoUHPLC-MS/MS) (Evosep One system coupled to timsTOF Pro mass spectrometer - Bruker Daltonics, Bremen, Germany), as extensively described in prior technical notes. ${ }^{24}$ Metabolomics analyses were performed as previously reported. ${ }^{25} 100 \mu \mathrm{L}$ of stored RBC were collected on a weekly basis, extracted at 1:6 dilution in methanol:acetonitrile:water (5:3:2) and analyzed by UHPLC-MS (Ultimate 3000 RSLC-Q Exactive, Thermo Fisher) (see the Online Supplementary Methods for details). Metabolite assignment was performed against an in house standard library, as reported, ${ }^{26}$ through the freely available software Maven (Princeton University, USA). No data pre-processing (neither normalization nor log-transformation) was performed.

\section{Statistical analysis}

For statistical analysis (SPSS Version 22.0, IBM Corp, NKUA) Shapiro-Wilk test and detrended normal $\mathrm{Q}-\mathrm{Q}$ plots (for testing normal distribution and outliers), independent $t$-test or repeated measures analysis of variance (ANOVA) with Bonferroni-like adjustment (for intergroup differences) and Pearson's or Spearman's tests (for correlation analysis) were used. Variables that exhibited repeatable correlations between each individual storage day (six time points) and fresh blood were used for the construction of in vivolex vivo biological networks (Cytoscape 3.7.2). Receiver operating characteristic (ROC) curves were used to find out parameters strongly indicative of $\beta \mathrm{Thal}^{+}$status in stored RBC. Significance was accepted at $P<0.05$.

\section{Results}

\section{Baseline features and storability of beta thalassemia red blood cells}

In a cohort of 204 eligible blood donors the $\beta \mathrm{Thal}^{+}$subgroup was approximately 9\%. As expected, lower $\mathrm{Hb}$ concentration and $\mathrm{RBC}$ indices but higher $\mathrm{RBC}$ count and $\mathrm{HbA}_{2}$ concentration were measured in $\beta \mathrm{Thal}^{+}$donors (ROC curve: area under the curve [AUC] for mean cor- 
puscular volume $[\mathrm{MCV}]=0.965$, for $\left.\mathrm{HbA}_{2}=1,000\right)$, who carried an array of $\beta-\mathrm{Hb}$ mutations commonly found in Greece (IVS I-1, IVS I-6, IVS I-110, IVS II-1 and IVS II745). While osmotic fragility was significantly lower in $\beta \mathrm{Thal}^{+} \mathrm{RBC}$, free $\mathrm{Hb}$ levels were similar to control. A trend for high total antioxidant capacity (TAC) and low plasma protein carbonylation was also observed in $\beta$ Thal $^{+}$ (Table 1).

For RBC storability analysis, the subgroup of $\beta \mathrm{Thal}^{+}$ donors $(n=18)$ was compared to an equivalent group of average controls $(n=20)$. The $\beta \mathrm{Thal}^{+}$units exhibited lower levels of free $\mathrm{Hb}$ (storage, oxidative, mechanical and osmotic hemolysis) either throughout the storage period (mechanically and osmotically induced hemolysis) or for the last 2 weeks of it (storage and oxidative hemolysis) (Figure 1A). According to ROC curve analysis, the osmotic, mechanical and storage hemolysis have very good potential to predict the $\beta \mathrm{Thal}^{+}$status at every time point of storage (Online Supplementary Figure S1A). Of note, only osmotic fragility (which follows a logarithmic increase during storage) was lower in the $\beta T$ hal ${ }^{+}$versus control RBC in vivo. Apart from hemolysis, the extracellular $\mathrm{K}^{+}$was also lower in $\beta \mathrm{Thal}^{+}$plasma and day 42 -supernatant (Figure 1B). Preliminary proteomics analysis revealed differences between the two groups in the abundance of membrane proteins physiologically related to the $\mathrm{RBC}$ volume control/cation homeostasis, including piezo-1, $\mathrm{Na}^{+} / \mathrm{K}^{+}$ATPase and aquaporin-1 (Figure $1 \mathrm{C}$ ).

Higher TAC and uric acid-dependent antioxidant capacities (UAdAC) were measured in the plasma/supernatant of the $\beta \mathrm{Thal}^{+}$samples compared to control (Figure $2 \mathrm{~A}$ ). ROC curve analysis revealed variations in both TAC and UAdAC, strongly indicative of the $\beta \mathrm{Thal}^{+}$status in stored RBC (Online Supplementary Figure S1B), in contrast to the uric acid-independent antioxidant capacity (UAiAC) levels that were very similar to control (Figure 2A). Membrane lipid peroxidation and protein carbonylation were significantly lower in the $\beta \mathrm{Thal}^{+}$versus control $\mathrm{RBC}$ throughout the storage period or from middle storage onwards, respectively (Figure $2 \mathrm{~B}$ ). Spontaneous intracellular ROS levels were quite similar between the two donor groups at early storage, but lower levels were detected in $\beta \mathrm{Thal}^{+}$versus control $\mathrm{RBC}$ in the late period (Figure 2C). Stress-induced (e.g., by tBHP, diamide, phenylhydrazine) ROS generation basically resulted in no between-group differences. Although significantly higher at baseline, the intracellular $\mathrm{Ca}^{2+}$ of $\beta \mathrm{Thal}^{+} \mathrm{RBC}$ exhibited only a trend toward higher levels during storage compared to controls (Figure 2D). In contrast, while similar at baseline (and for the first weeks of storage), PS externalization was significantly lower in $\beta \mathrm{Thal}^{+} \mathrm{RBC}$ for the last 2 weeks (Figure 2E). According to a preliminary proteomics analysis, the membrane expression of calcium related proteins (such as calmodulin, calpain and annexin A7/synexin) and of the lipid remodeling protein scramblase differed significantly between the two groups of stored RBC (Figure 2F).

\section{Metabolic profile of beta thalassemia red blood cells in vivo and during storage in CPD-SAGM}

Full metabolomics analyses were performed in paired fresh and stored samples from the control and $\beta \mathrm{Thal}^{+}$ groups ( $\mathrm{n}=15$, Online Supplementary Figures $S 2$ to $S 11)$. In the case of fresh RBC (Figure $3 \mathrm{~A}$ ) partial least square-discriminant analysis (PLS-DA) separated the two groups across principal component 1 (PC1), explaining approximately $11 \%$ of the total variance (Figure $3 \mathrm{~B}$ ). The top 25 metabolic changes between the two groups - as determined by $t$-test - are highlighted in the heat map in Figure $3 \mathrm{C}$. Increases were observed in $\beta \mathrm{Thal}^{+} \mathrm{RBC}$ with respect to metabolites derived from glycolysis or branching pathways (2,3-diphosphoglycerate, phosphoglycerate isomers, phosphoenolpyruvate) and PPP (6-phosphogluconate, ribose mono and diphosphate, NADPH) (Figure 3C). Overall, these steady state analyses are suggestive of activation of PPP and glycolysis upstream to pyruvate kinase in fresh $\beta \mathrm{Thal}^{+} \mathrm{RBC}$, in the absence of significant alterations of glutathione pools and turn-over (Figure $4 \mathrm{~A}$ to $C$, respectively). Indeed, $\beta T_{h a l^{+}} \mathrm{RBC}$ were characterized by decreases in pyruvate, arginine, creatine, glycine, inosine and monophosphate (Figure 3C), suggestive of alterations in purine deamination/oxidation and arginine/polyamine metabolism (Figure 4D to E).

Metabolomics analyses were thus performed on stored RBC units from the same donors on a weekly basis (Figure 3D). Unsupervised principal component analysis (PCA) and hierarchical clustering analysis of significant metabolites by repeated measures ANOVA are shown in

Table 1. Selected characteristics of beta thalassemia blood minor in vivo compared to control.

\begin{tabular}{|c|c|c|}
\hline & Control (n=186) & $\beta$ Thal+ $(n=18)$ \\
\hline Red blood cells (x106/ $/ \mathrm{L})$ & $4.94 \pm 0.33$ & $5.88 \pm 0.34^{*}$ \\
\hline Hematocrit (\%) & $44.07 \pm 2.58$ & $39.84 \pm 2.20^{5}$ \\
\hline Total Hemoglobin (g/dL) & $15.00 \pm 1.07$ & $13.36 \pm 0.71^{*}$ \\
\hline Mean corpuscular volume (fL) & $89.38 \pm 4.17$ & $67.07 \pm 6.72^{*}$ \\
\hline Mean corpuscular hemoglobin (pg) & $30.44 \pm 2.08$ & $22.51 \pm 2.42^{*}$ \\
\hline Mean corpuscular hemoglobin concentration (g/dL) & $34.00 \pm 1.09$ & $32.69 \pm 0.67^{s}$ \\
\hline Red cell distribution width (\%) & $12.48 \pm 0.57$ & $13.64 \pm 0.88$ \\
\hline Mean platelet volume (fL) & $7.47 \pm 1.57$ & $9.24 \pm 2.17^{8}$ \\
\hline Mean corpuscular fragility $(\%[\mathrm{NaCl}])$ & $0.438 \pm 0.025$ & $0.389 \pm 0.028^{*}$ \\
\hline Free hemoglobin (mg/dL) & $3.61 \pm 1.83$ & $2.73 \pm 1.24$ \\
\hline Total antioxidant capacity of plasma (TAC) $\left(\mu \mathrm{M} \mathrm{Fe}^{2+}\right)$ & $622 \pm 94.2$ & $735 \pm 142^{\$}$ \\
\hline Carbonylated proteins of plasma (nmol/mg) & $0.350 \pm 0.107$ & $0.296 \pm 0.111^{s}$ \\
\hline
\end{tabular}

Data are presented as mean \pm standard deviation. ${ }^{*} P<0.05 ;\left({ }^{\S}\right)$ marginal significance. $\beta$ Thal': beta thalassemia minor. 
Figure $3 \mathrm{E}$ and $\mathrm{F}$, respectively. Of note, several of the metabolic differences between the two groups at baseline were further exacerbated during storage, with significantly lower levels of IMP, hypoxanthine and adenosine monophosphate (AMP) in the stored $\beta \mathrm{Thal}^{+} \mathrm{RBC}$ (Figure $5 A)$. On the other hand, the $\beta \mathrm{Thal}^{+} \mathrm{RBC}$ had higher levels of urate throughout storage. Since human RBC lack a functional uricase, chemical oxidation of urate promotes further catabolism to allantoin and allantoate, that were found significantly lower and higher in the $\beta T h \mathrm{I}^{+} \mathrm{RBC}$, respectively, throughout storage (Figure 5A). These observations were accompanied by a higher degree of glutamine consumption and glutamate generation in $\beta$ Thal $^{+}$RBC after storage day 7 (Figure 5A; Online Supplementary Figure S8, respectively), suggestive of ongoing glutaminolysis. Interestingly, metabolites of purine metabolism such as urate, allantoate and glutamine represent very good predictors of $\beta \mathrm{Thal}^{+}$status in stored RBC (ROC curve analysis, Online Supplementary Figure S1C). Intertwined with purine (especially adenosine) metabolism, S-adenosylmethionine (SAM) synthesis and consumption were not significantly altered in

\section{$\beta_{\text {Thal }}{ }^{+}$Control}

A
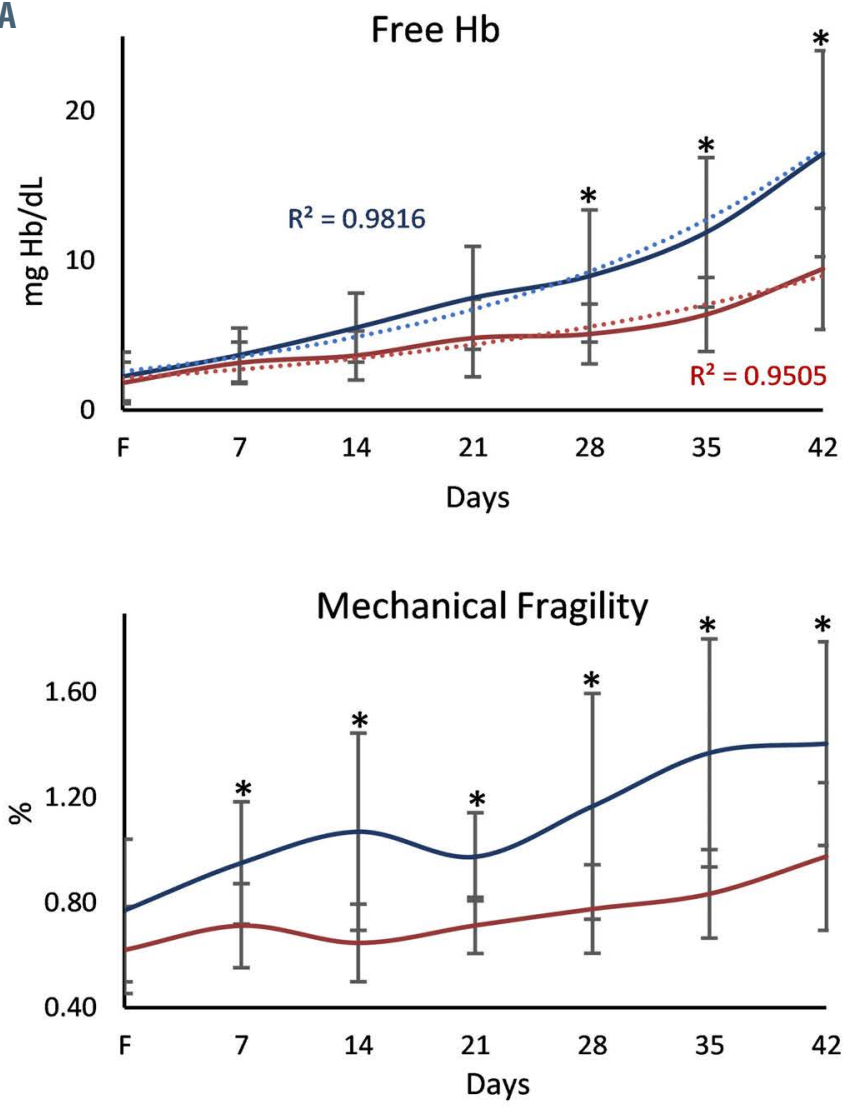

B

C
Piezo-1
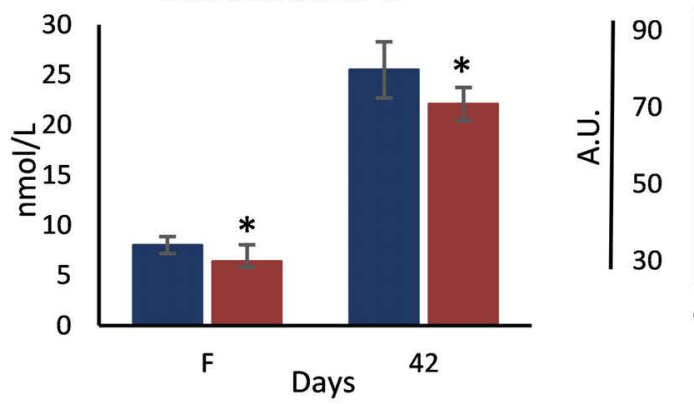

Osmotic Fragility

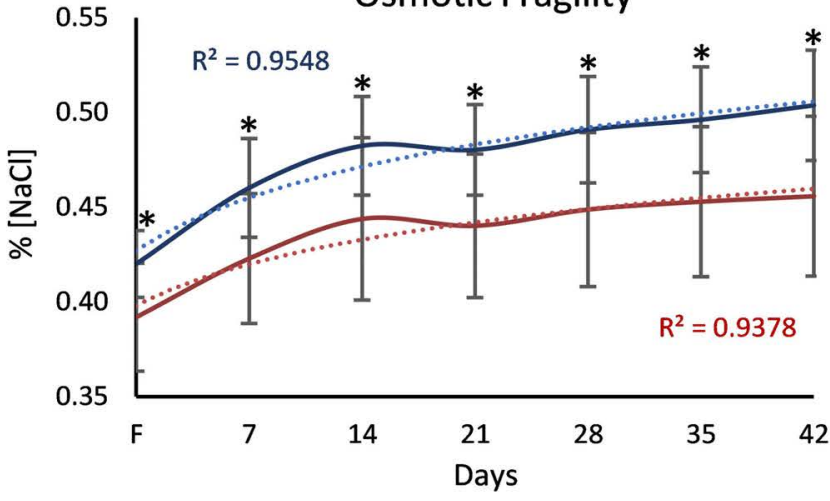

Oxidative Haemolysis

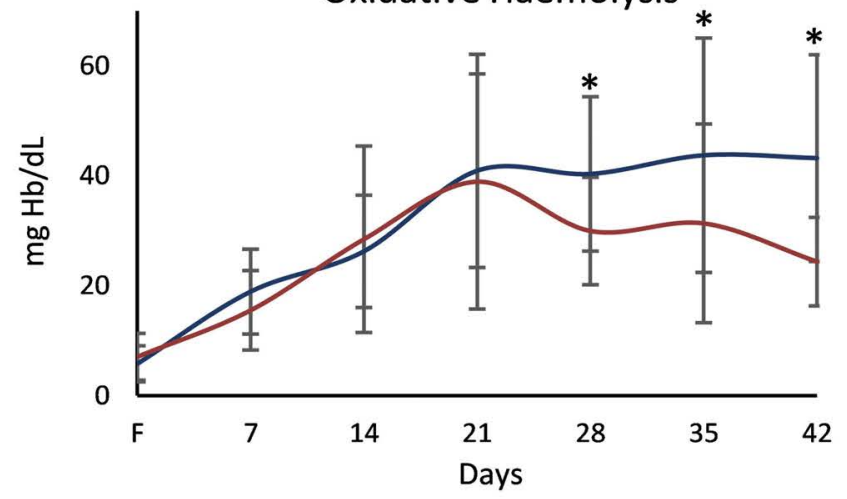

$\mathrm{Na}^{+} / \mathrm{K}^{+}$ATPase

Aquaporin-1

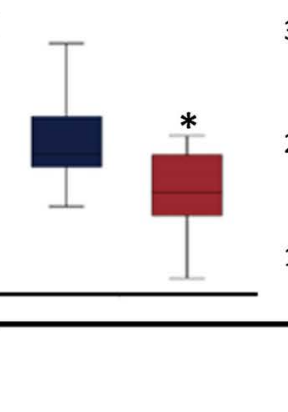

35

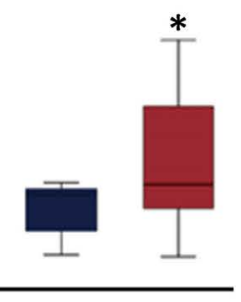

160

120

80

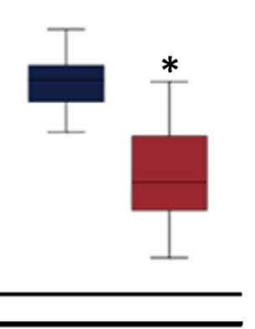

Storage Levels

Figure 1. Variation in hemolysis variables of fresh and stored red blood cells in beta thalassemia minor and control donors. (A) Time course evaluation of storage, osmotic, mechanical and oxidative hemolysis. Dashed lines represent trend lines of exponential or logarithmic models for storage and osmotic hemolysis, respectively. (B) Potassium leakage. (C) Storage levels of selected proteins related to osmotic/mechanical hemolysis. Data are presented as mean \pm standard deviation. $\star P<0.05$; F: fresh blood; A.U.: arbitrary units; $\beta$ Thal': beta thalassemia minor. 
A
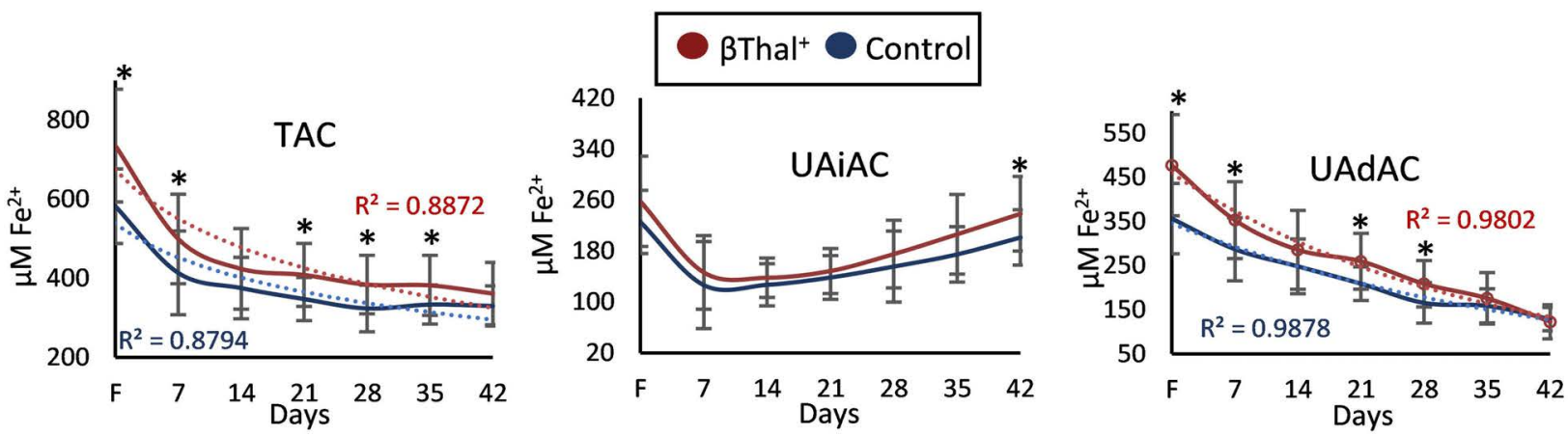

B
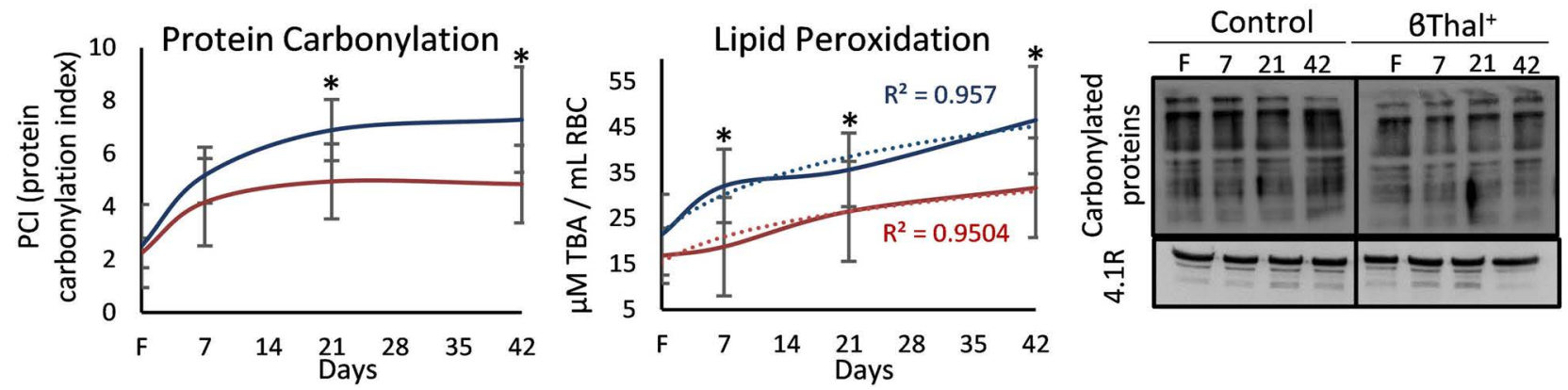

C
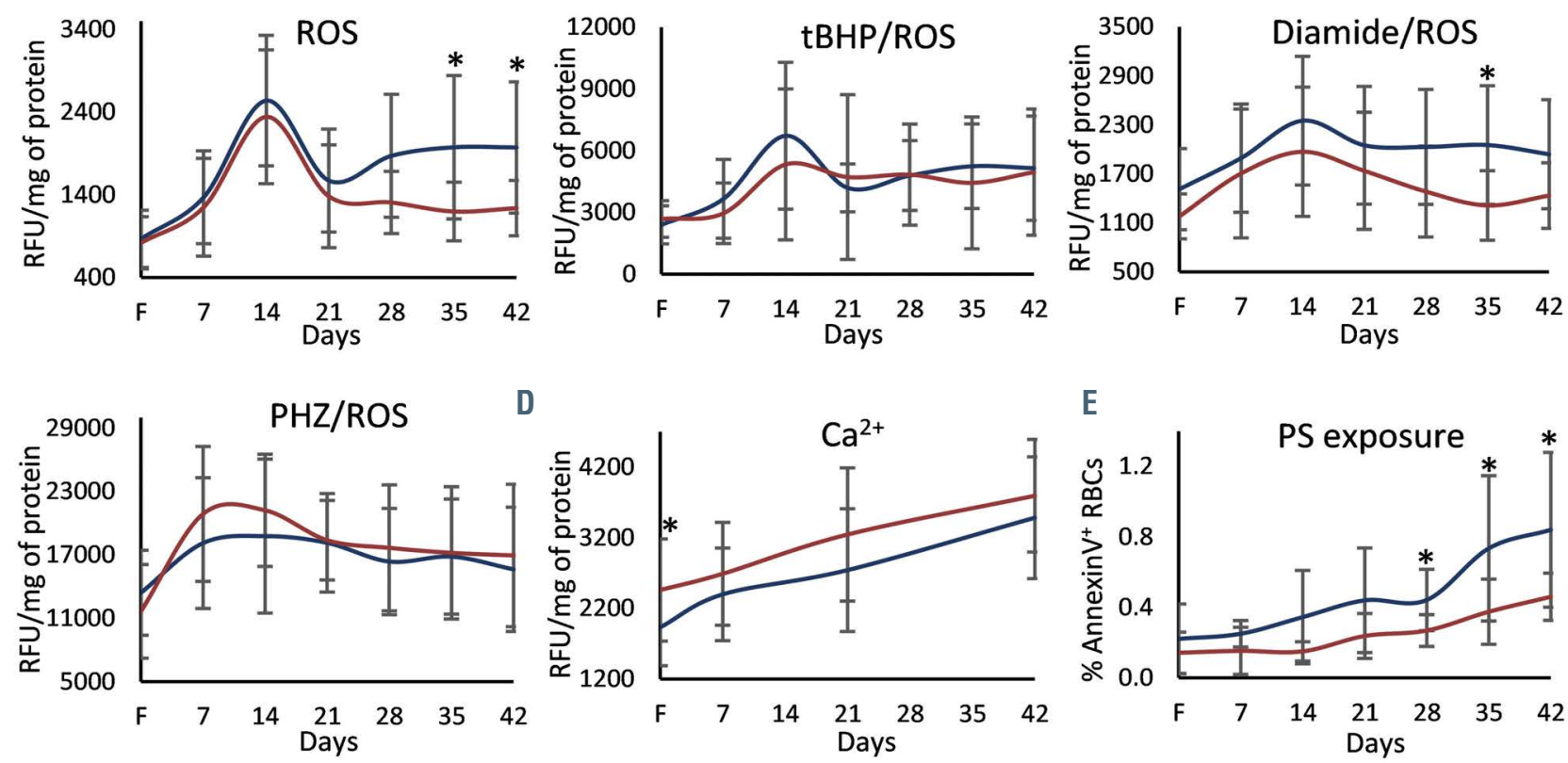

E

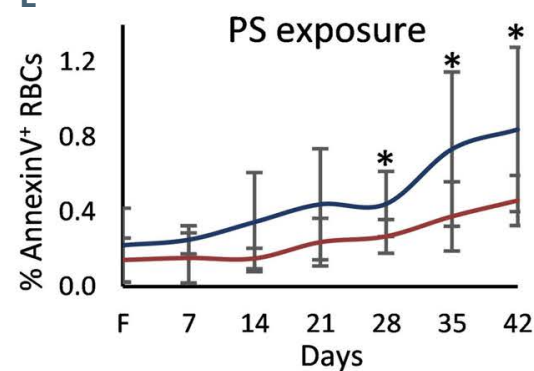

$\mathbf{F}$

Calmodulin-1

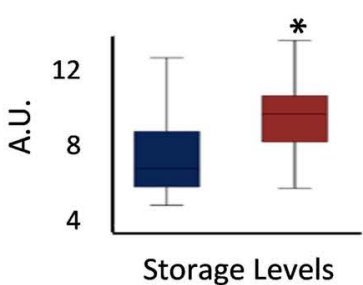

Calpain-1

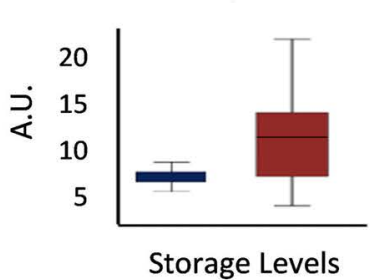

Annexin A7

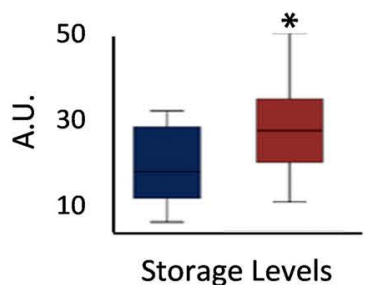

Scramblase

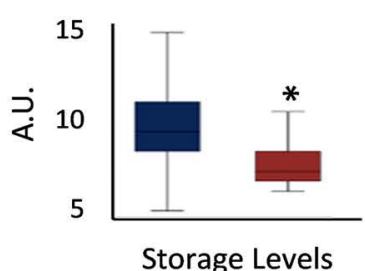

Figure 2. Redox status and calcium signaling of red blood cells from beta thalassemia minor and control donors. (A) Time course variation of total (TAC), uric acidindependent (UAAC) and uric acid- dependent (UAdAC) antioxidant capacity. (B) Membrane lipid peroxidation and protein carbonylation ( $\mathrm{n}=8$ per group; $4.1 \mathrm{R}$ protein was used for internal control). (C) Variation of endogenous/exogenously induced (tert-butyl-hyperoxide; tBHP, diamide and phenylhydrazine; PHZ) reactive oxygen species (ROS) levels. (D) Intracellular calcium accumulation. (E) Phosphatidylserine (PS) exposure. (F) Storage levels of selected proteins related to calcium homeostasis and lipid remodeling. Dashed lines represent trendlines of logarithmic models. Data are presented as mean \pm standard deviation. $* P<0.05$; F: fresh blood; TBA: thiobarbituric acid; $\beta$ Thal $^{+}$: beta thalassemia minor. 
$\mathrm{RBC}$ from the $\beta \mathrm{Thal}^{+}$group (Figure $5 \mathrm{~B}$ ). Cysteine levels were higher and cysteate levels lower in the $\beta \mathrm{Thal}^{+}$ group, especially past storage day 28 and at the end of storage (Figure 5B). Significantly higher levels of sphingosine 1-phosphate (S1P) - a marker of systemic hypoxemia $^{27}$ - were observed in RBC from the $\beta T_{\text {Thal }}{ }^{+}$group on day 0 and throughout storage (Figure 5C).

Like in fresh RBC, changes were observed in arginine metabolism between the two groups as a result of storage duration - with RBC from the $\beta \mathrm{Thal}^{+}$group being charac- terized by significantly lower arginine and creatine and higher asymmetric dimethyl-arginine and acetyl-spermidine levels throughout storage (Figure 6A). Of all the pathways assessed in this study, the most notable betweengroup changes were unexpectedly found in vitamin B6 metabolism (Figure $6 \mathrm{~B}$ ) and in the hexosamine pathway (Figure $6 \mathrm{C}$ ). $\beta \mathrm{Thal}^{+} \mathrm{RBC}$ were characterized by lower pyridoxate and pyridoxal and higher pyridoxamine levels throughout storage. On the other hand, all intermediates of the hexosamine pathway (uridine monophosphate -

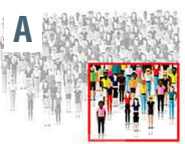

Control $(n=13)$ $\beta$ Thalassemia Heterozygotes $(\mathrm{n}=12)$

B PLS-DA Day 0

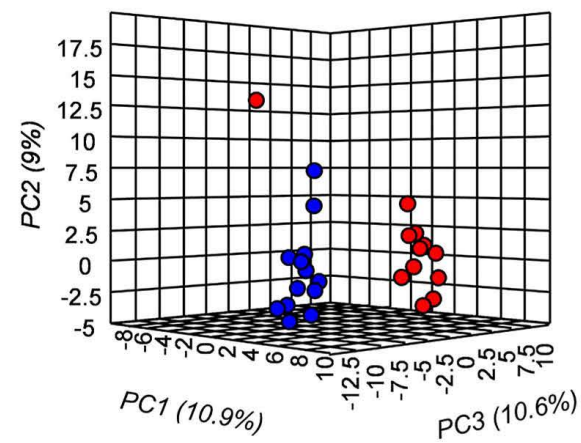

Groups

Control $\beta$ Thal $^{+}$

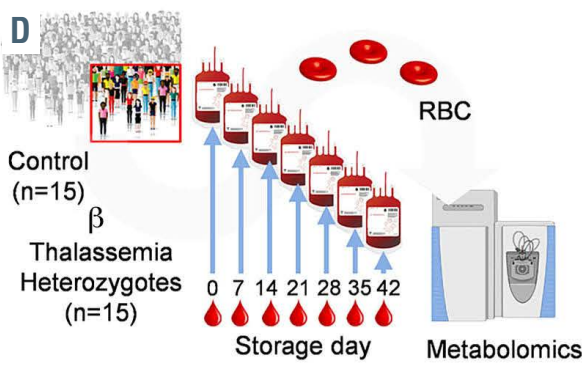

E
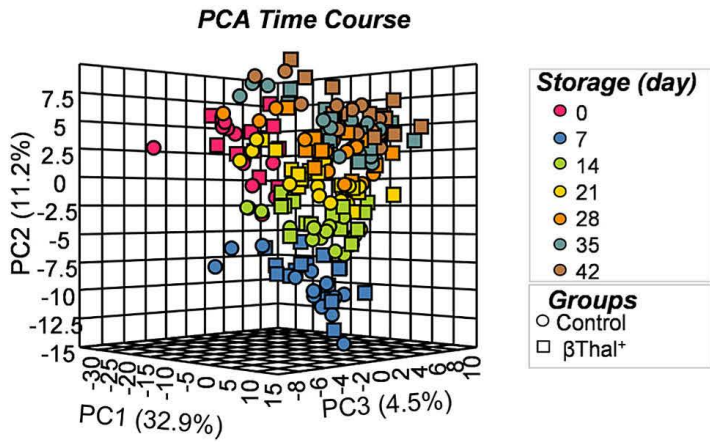

C Control $\quad \beta$ Thal $^{+}$

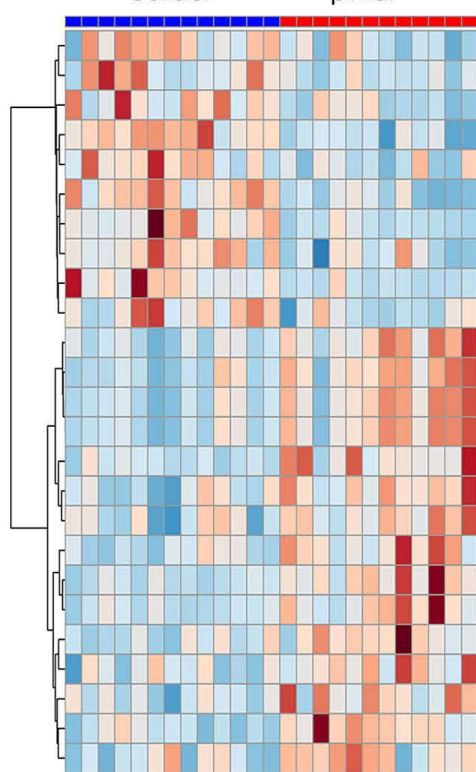

-arginine

Pantetheine UDP-D-glucuronate

Creatine glycine 5-Methylthioadenos IMP Nicotinic acid UDP-acetyl-glucosamine Pyruvate 2/3P-glycerate 2,3-Bisphosphoglycerate Phosphoenolpyruvat 3-Phosphonopyruvat Guanine D-Ribose $1 P$ D-Ribose 5DP Spermidine $\mathrm{N}$-acetyl-glucosamine Glucosaminine Indole-3-acetate Asymmetric dimethylarg 6P-Gluconate Pyridoxamine NADPH BThal $^{+}$
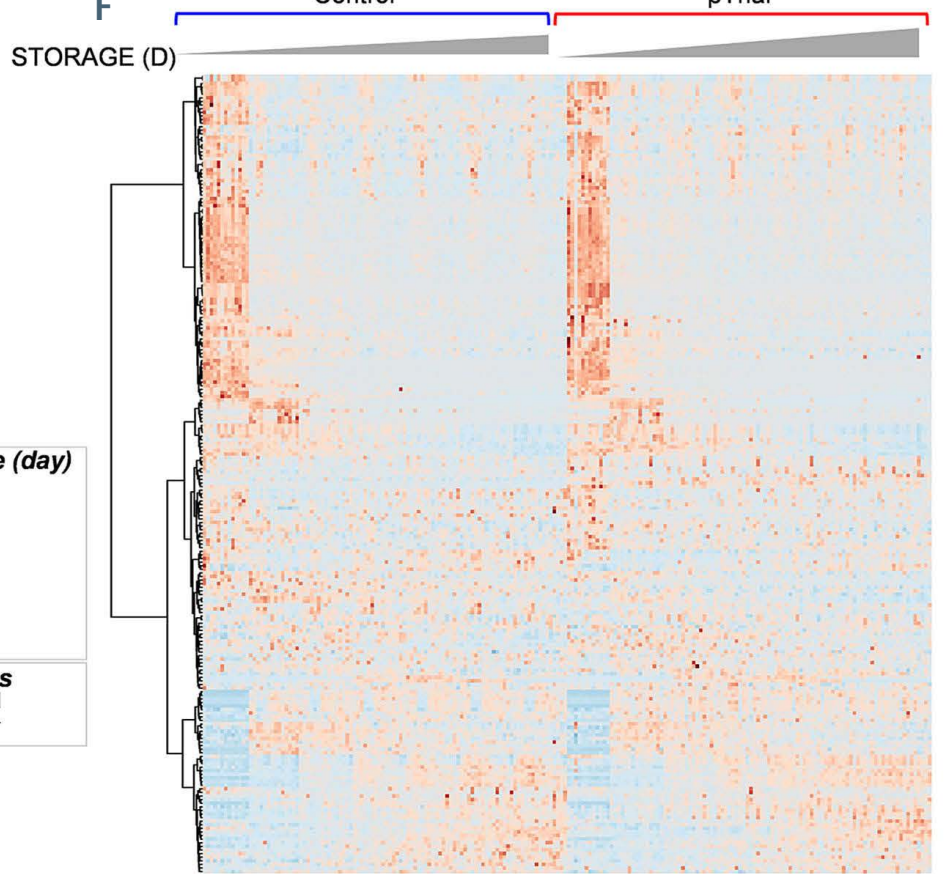

Figure 3. Metabolomics analyses of fresh and stored red blood cells from control and beta thalassemia minor donors. (A) Fresh blood analysis of eligible blood donors ( $n=13$ for controls and $n=12$ for beta thalassemia minor [ $\beta$ thal $\left.{ }^{+}\right]$donors). (B) Principal component and discriminant analysis in fresh blood. (C) Heat map of top 25 metabolites differing between $\beta$ thal $^{+}$and control fresh red blood cells (RBC). (D) Metabolomics analysis was performed in RBC stored in leukoreduced CPD/SAGM units ( $n=15$ per group). (E) Principal component and discriminant analysis in stored RBC. (F) Heat map of variation in RBC metabolites throughout storage. CPD/SAGM: citrate-phosphate-dextrose/saline-adenine-glucose-mannitol. 
UMP, acetyl-glucosamine, acetyl-glucosamine phosphate) were significantly higher in $\beta \mathrm{Thal}^{+} \mathrm{RBC}$ at all storage time points, while the final product of this pathway - uridine diphosphate (UDP) $N$-acetyl-glucosamine was significantly lower in $\beta$ Thal $^{+}$RBC (Figure $6 \mathrm{C}$ ), suggestive of either a blockade at the metabolic step catalyzed by UDP-Nacetylglucosamine phosphorylase or an increased consumption of its product for protein O-GlcNAcylation (post-translational attachment of $O$-linked $N$-acetylglucosamine moieties to serine and threonine residues).
A
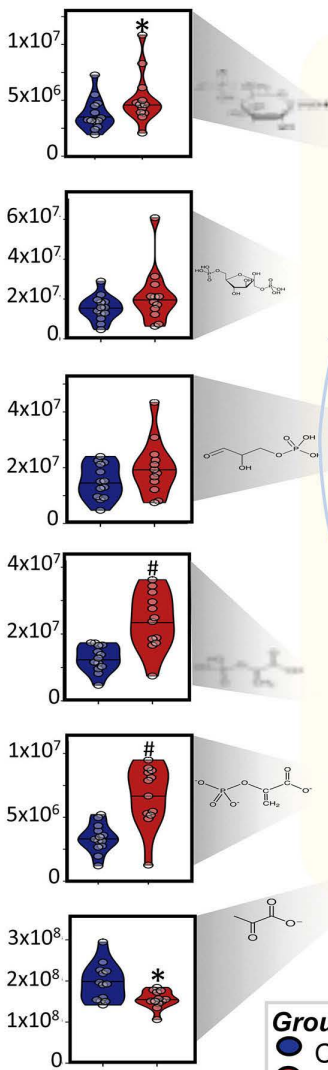

D

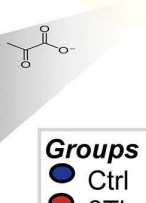

ß BThal $^{+}$

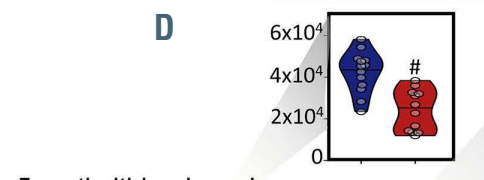

5-methylthioadenosine

$$
\text { SAM NAD }{ }^{+}
$$

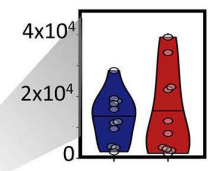

NADH

Malate
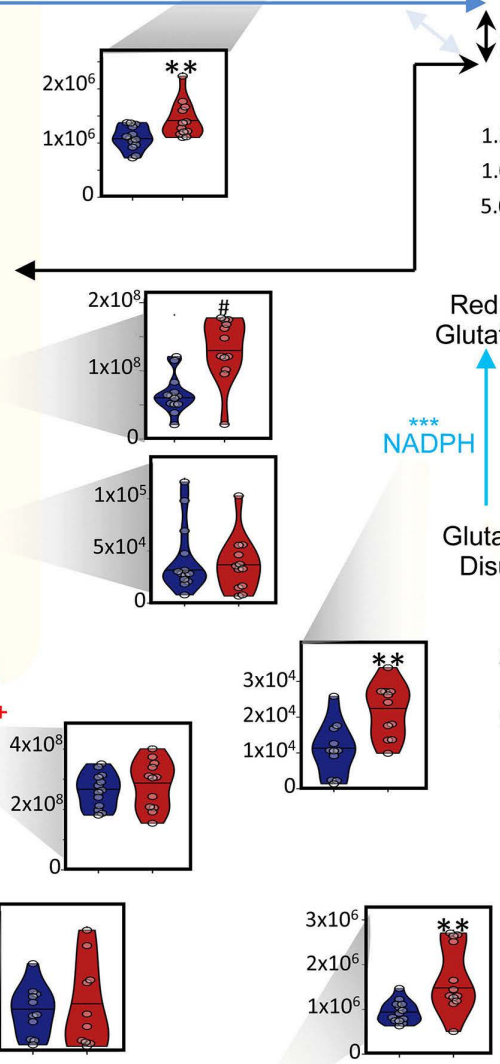

Guanine Guanosine

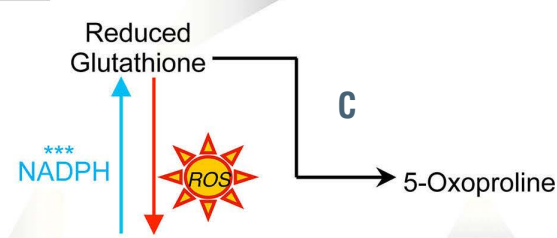

Glutathione Disulfide
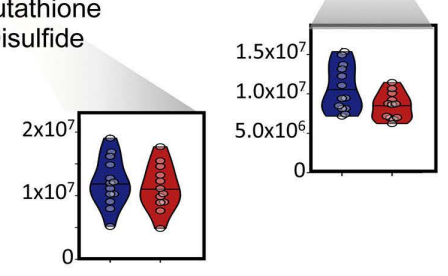

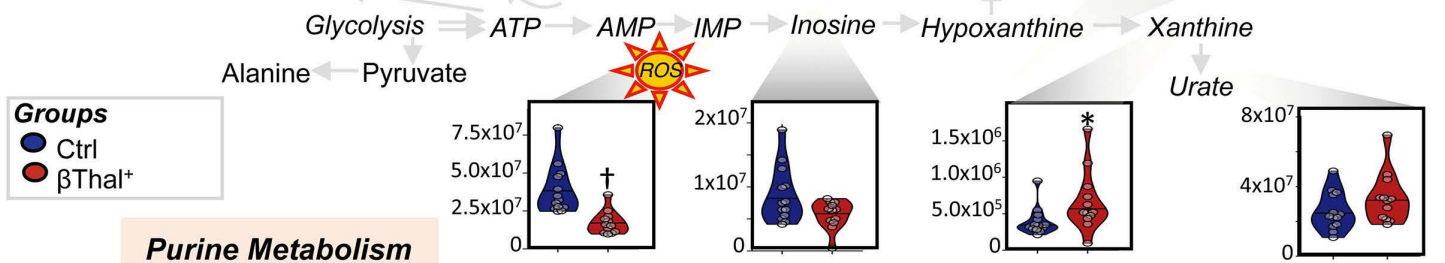

E

Arginine Metabolism

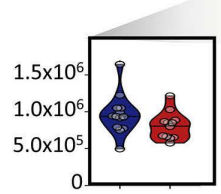

Citrulline
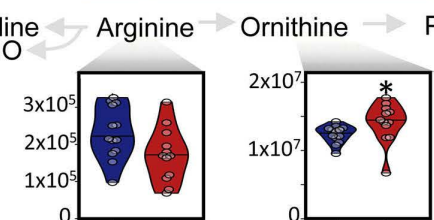

Putrescine Spermidine
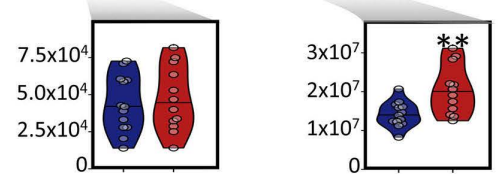
Again, ROC curve analysis highlighted the potential of glucosamines to distinguish stored $\beta \mathrm{Thal}^{+} \mathrm{RBC}$ from their control counterparts (Online Supplementary Figure S1D). Finally, RBC from $\beta \mathrm{Thal}^{+}$donors were characterized by higher levels of carnosine and indole-acetate - metabolites with either antioxidant activity or associated microbial metabolism, respectively, from baseline throughout storage, with no storage-dependent fluctuations, suggestive of differences in the circulating blood metabolome between the two groups at the time of donation (Figure 6D).

A

Purine Metabolism

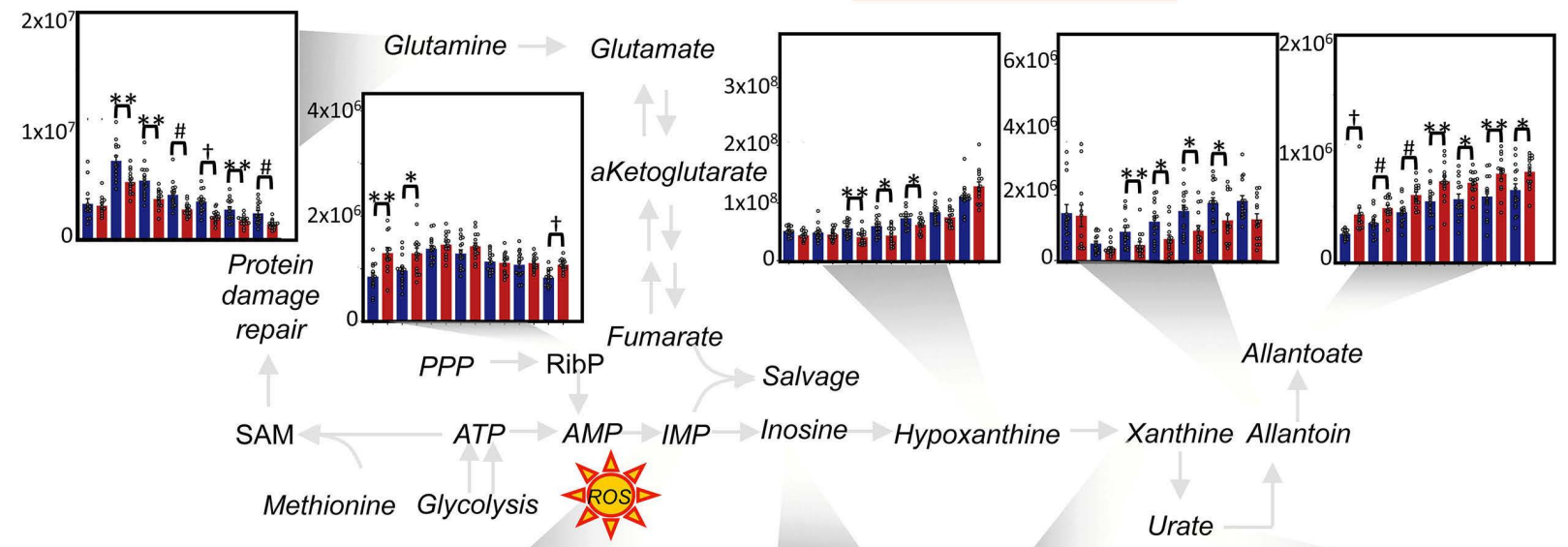

B
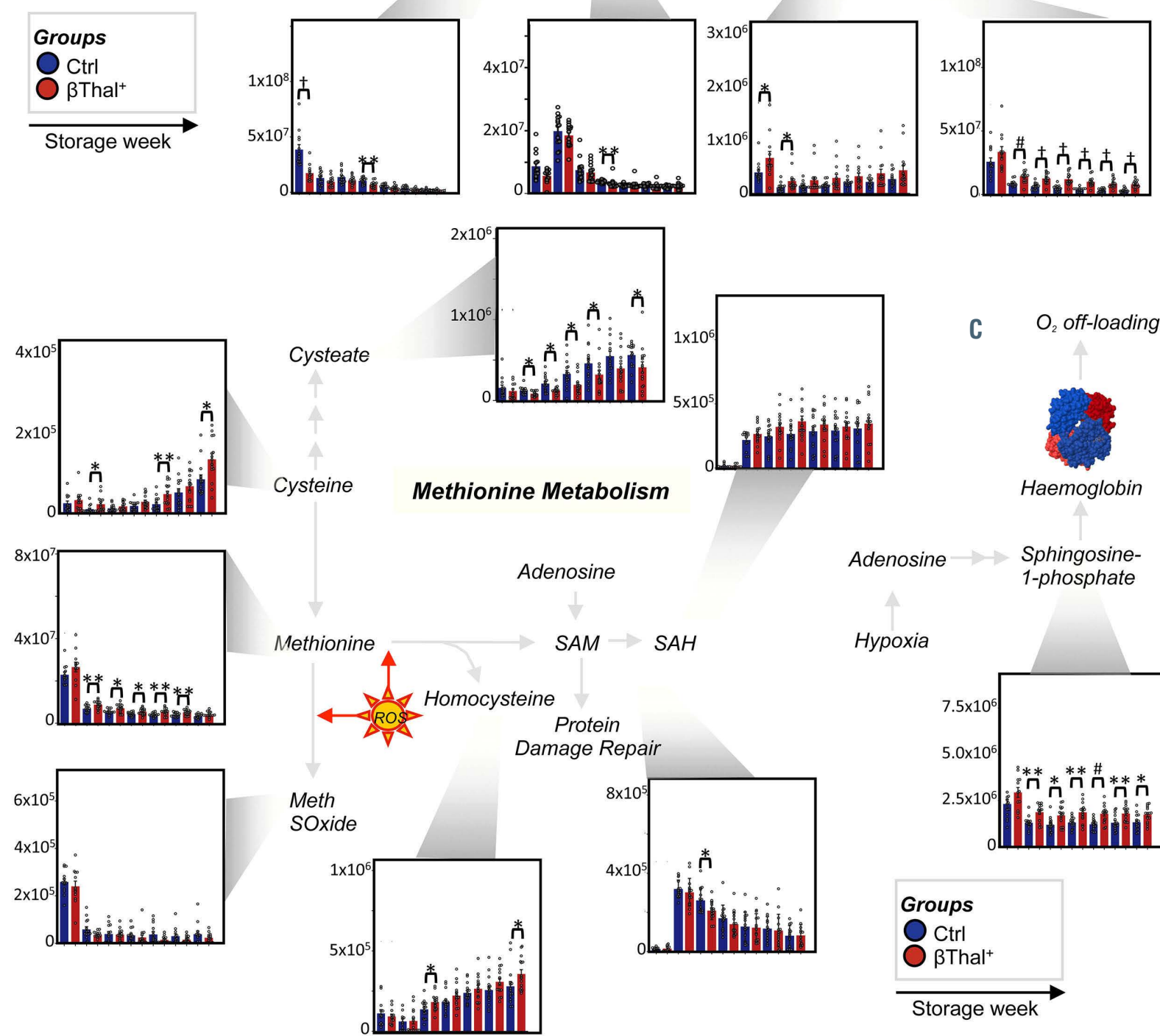

Hypoxia
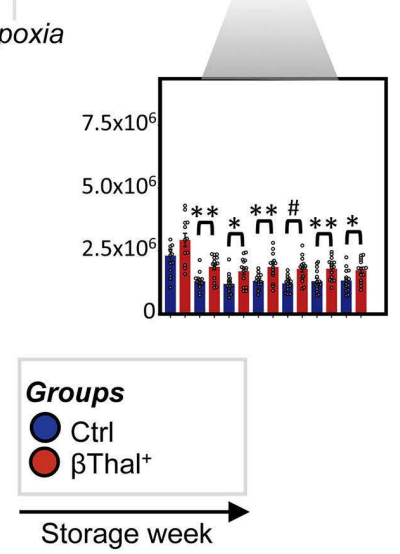

Figure 5. Distinct metabolic pathways in beta thalassemia minor donors during storage I. (A) Purine metabolism. (B) Methionine metabolism. (C) Hypoxia modulated hemoglobin loading via a sphingosine-1-phosphate effect. SAM: S-adenosyl methionine; SAH: S-adenosyl homocysteine; RibP: ribose phosphate. $\star P<0.05$, $* * P<0.01,{ }^{\sharp} P<0.001,{ }^{\dagger} P<0.0001$. 
Intertwining biological networks in stored red blood cells and supernatants

In order to find out major aspects of $\beta \mathrm{Thal}^{+} \mathrm{RBC}$ physiology, able to interconnect the in vivolex vivo states, and thus, to reveal the "effect" (if any) of baseline variables upon storage, we proceeded to comparative $\beta$ Thal $^{+}$versus control in vivolex vivo network analysis.

Our findings highlighted osmotic hemolysis as a "hub" parameter in $\beta \mathrm{Thal}^{+}$networks, with significant differences in the type and the amount of connections between
A
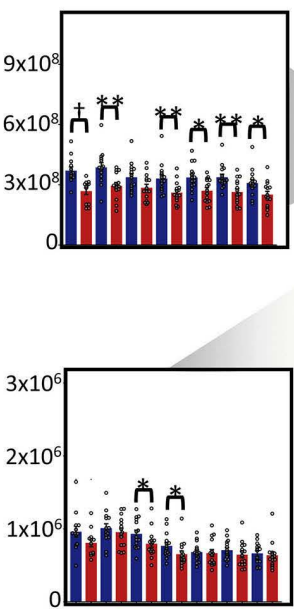

Arginine Metabolism

SAM $\rightarrow$ Methylated Protein Arginine
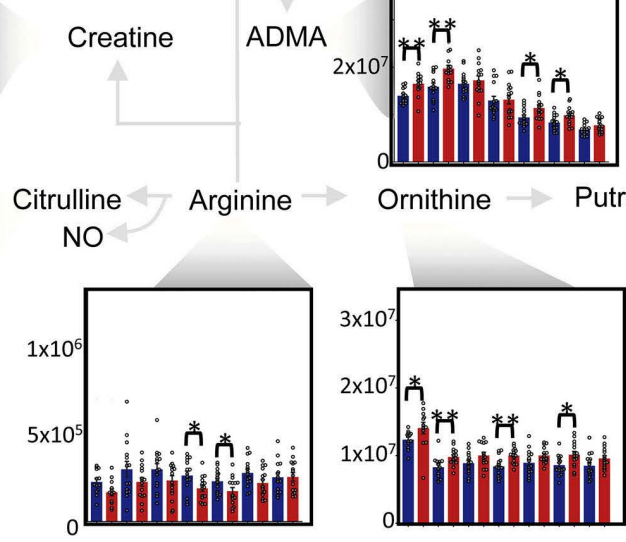

Ornithine $\rightarrow$ Putrescine

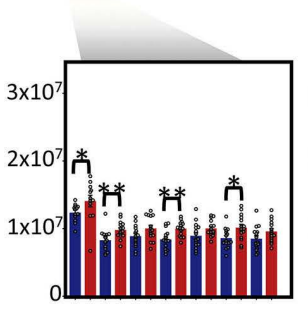

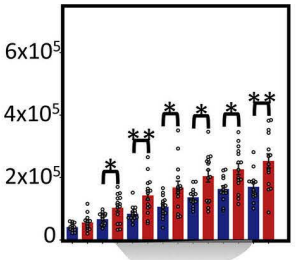

Acetyl-Spermidine
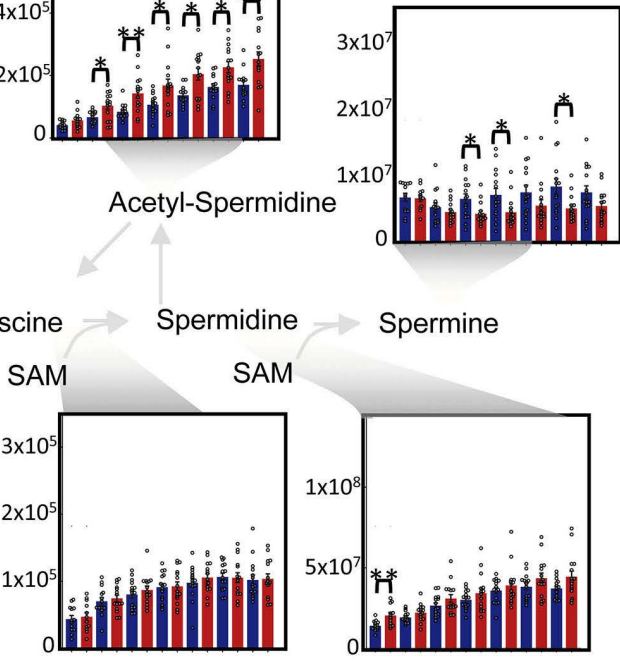

Spermine

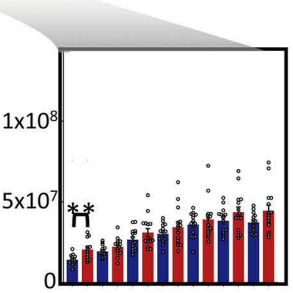

B

Pyridoxate

Pyridoxal $P$

Pyridoxamine
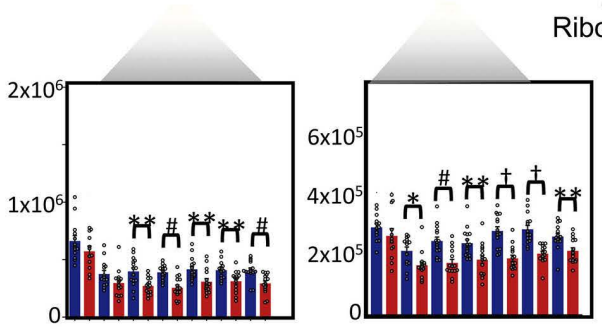

Riboflavin

Vitamin B6 Metabolism

C
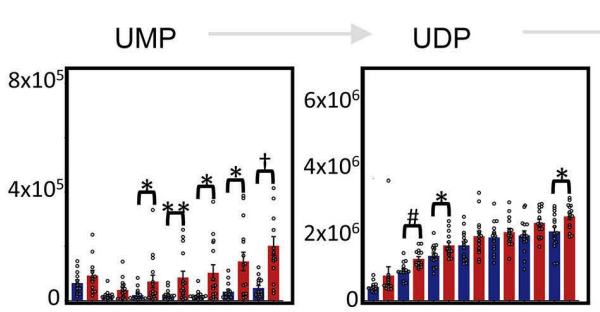

UDP-N-acetyl-

Glucosamine
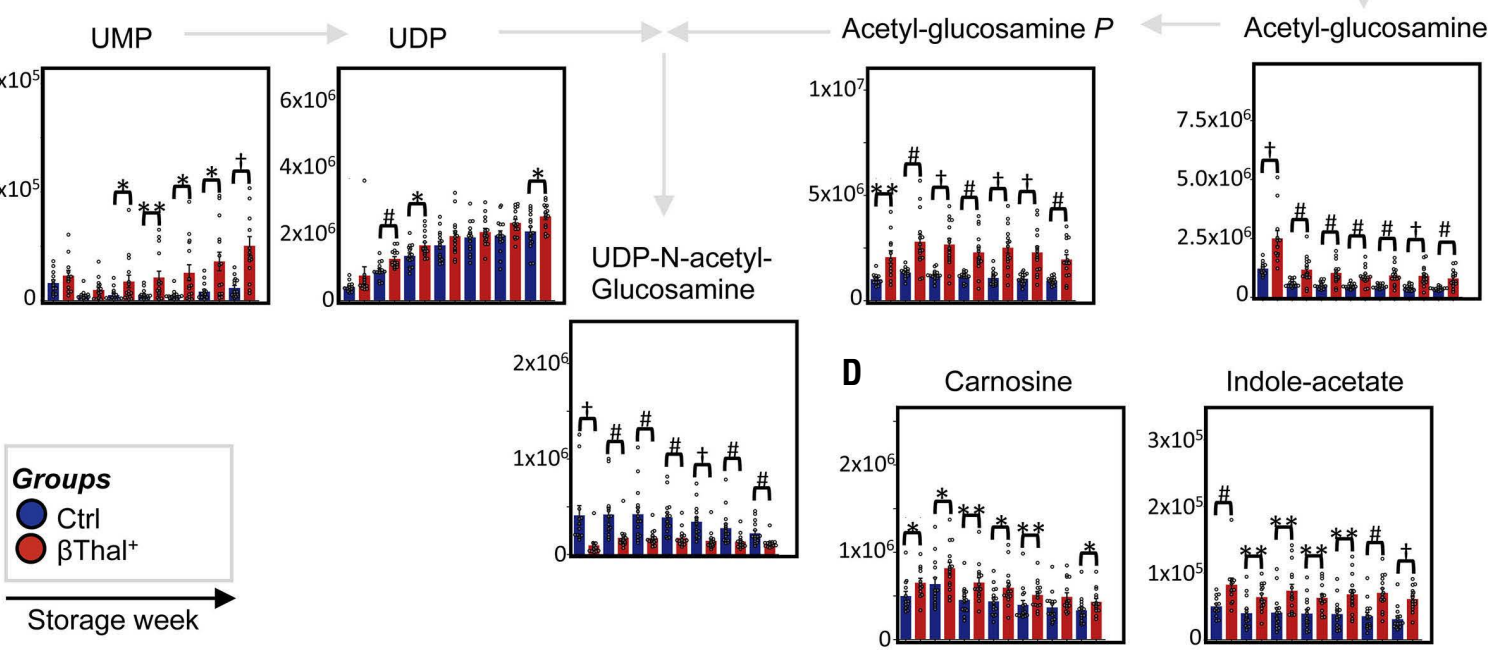

Figure 6. Distinct metabolic pathways in beta thalassemia minor donors during storage II. (A) Arginine metabolism. (B) Vitamin B6 metabolism. (C) Hexosamine pathway. (D) Carnosine and indole-acetate. SAM: S-adenosyl methionine; ADMA: asymmetric dimethylarginine; ${ }^{*} P<0.05, * * P<0.01,{ }^{*} P<0.001,{ }^{\dagger} P<0.0001$. 
A

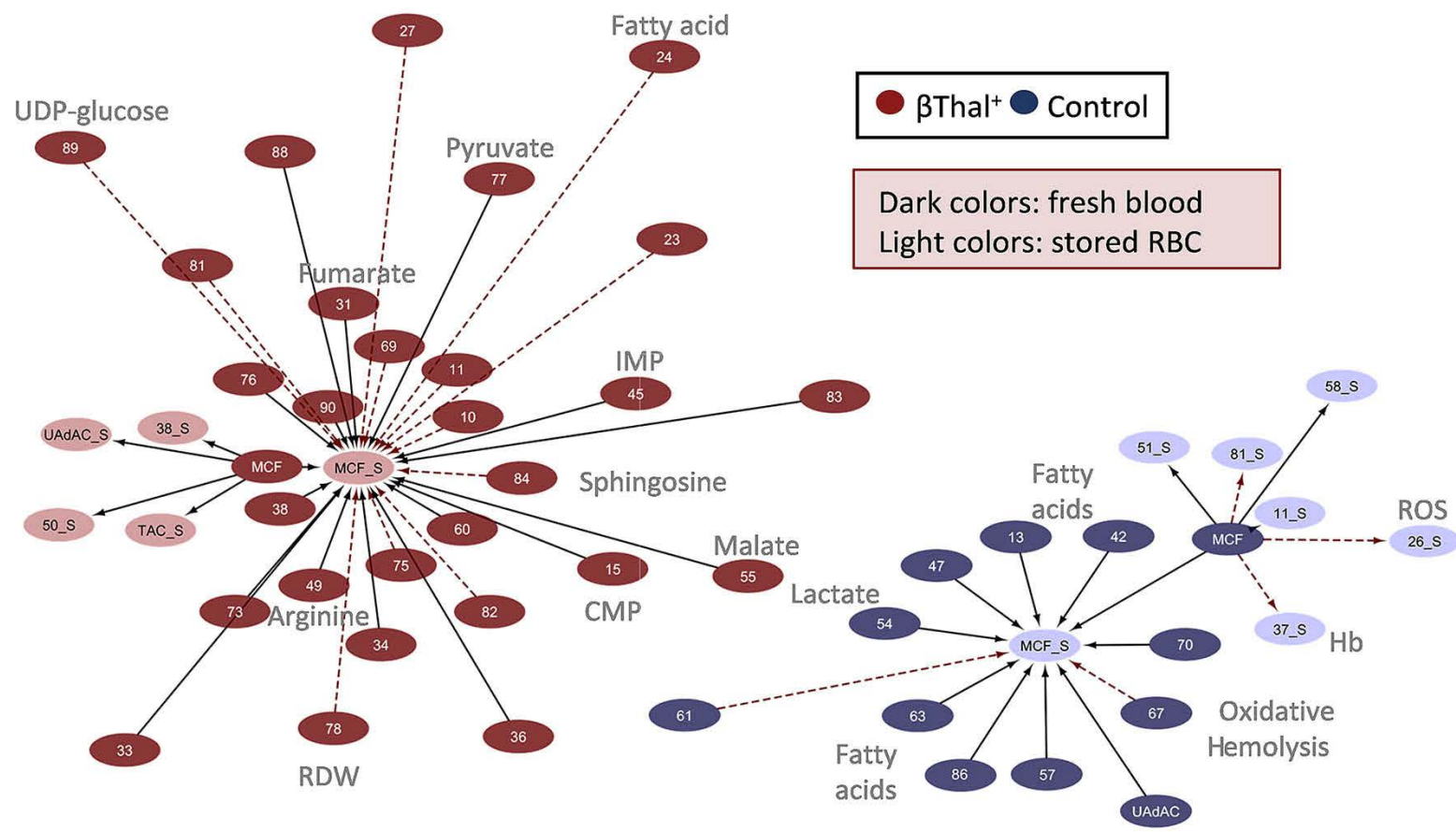

B

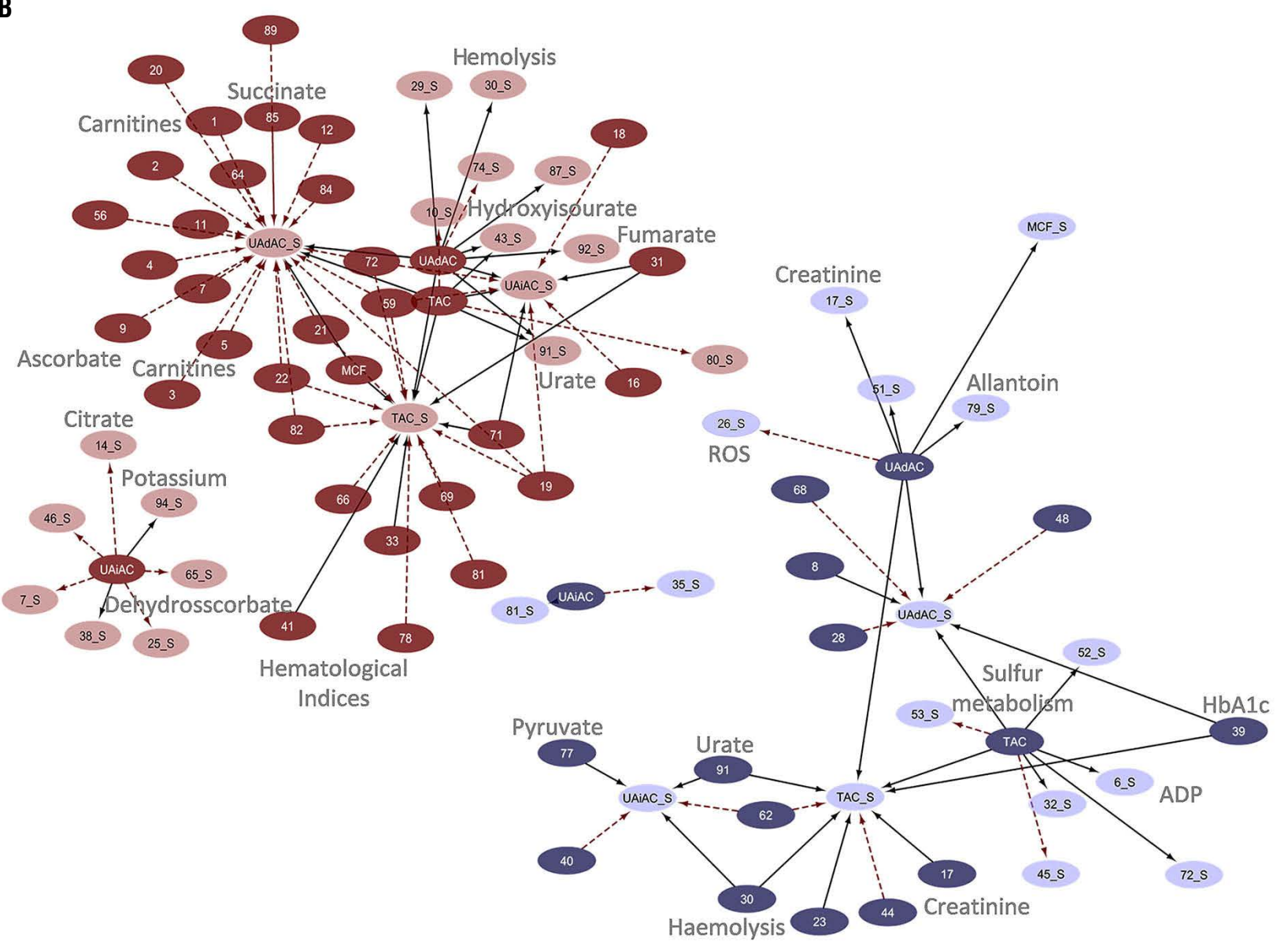

Figure 7. Network analysis of statistically significant repeated correlations between parameters of fresh blood and stored red blood cells. (A) Osmotic fragility hub. (B) Plasma/supernatant antioxidant activity hub. Solid black lines: positive correlation; dashed red lines: negative correlation. MCF: mean corpuscular fragility; TAC/UAdAC/UAiAC: total, uric acid dependent and uric acid independent antioxidant capacities. Numbers correspond to the definitions provided in the Online Supplementary Table S2. 
the groups. The osmotic fragility of stored $\beta T_{h a l^{+}} \mathrm{RBC}$ exhibited strong correlations with several biochemical (e.g., transferrin, $\mathrm{r}=0.560, P<0.01$ ), cellular (e.g., $\mathrm{RBC}$ mechanical hemolysis, $\mathrm{r}=0.771, P<0.01)$, protein and metabolic (e.g., L-arginine, $\mathrm{r}=0.697$, sphingosine-1-phosphate, $r=-0.636, P<0.01)$ parameters of $\beta \mathrm{Thal}^{+} \mathrm{RBC}$ and plasma, in striking contrast to the controls (Figure 7A). Notably, in the case of $\beta \mathrm{Thal}^{+} \mathrm{RBC}$, fragility variation during storage was less correlated to the in vivo levels of fatty acids but more correlated to those of carboxylic acids, compared to control. Despite the above-mentioned differences, osmotic hemolysis during storage was found interrelated with the in vivo levels of $\mathrm{RBC}$ aging markers and energy metabolism in both donor groups.

In similarity to the MCF network, the extracellular antioxidant capacity of the $\beta T_{\text {Thal }}^{+}$units presented higher connectivity with baseline parameters compared to control (twice the number of connections) including several metabolites of energy metabolism (e.g., Dglucose/UAdAC, $\mathrm{r}=-0.705, P<0.01)$, purine oxidation (e.g., TAC/5-hydroxyisourate, $\mathrm{r}=0.756, P<0.01$ ), urea cycle (e.g., ornithine/UAdAC, $\mathrm{r}=-0.769, P<0.01$ ) and carnitines (Figure $7 \mathrm{~B}$ ). Those findings suggested that that the basic physiology of $\beta T_{h a l^{+}}$is strongly and intrinsically related with the unique resistance to osmotic hemolysis and the high antioxidant capacity of the RBC units throughout storage.

\section{Discussion}

According to an increasing number of studies, genetic diversity among donors substantially affects RBC storage stability. Results of the REDS-III-Omics study showed that female sex and African American or Asian race-ethnicity $^{2}$ predispose to low susceptibility to storage and stress hemolysis. RBC from G6PD-deficient donors exhibit control levels of storage hemolysis, opposite to sickle cell trait RBC that are more susceptible to spontaneous hemolysis during storage. ${ }^{28}$ Our study reports for the first time that $\beta$ Thal ${ }^{+} \mathrm{RBC}$ are superior to those of the general population in terms of all hemolysis measures (including the less studied mechanical one), either throughout the storage period or for the more susceptible last 2 weeks of it. Superior secondary metrics of storage quality (including removal signaling and extracellular potassium) were also detected in $\beta T h a l^{+}$. As expected, the unique storage capacity of $\beta \mathrm{Thal}^{+} \mathrm{RBC}$ was found mechanistically linked to changes in specific metabolic flows and membrane protein expression profiles.

A tentative explanation for resistance to hemolysis lies in the appreciation of decreased HCT, MCH and Hb levels in $\beta \mathrm{Thal}^{+}$. Similar findings in high frequency blood donors have been associated with decreases in ferritin and total iron pools and improved hemolytic parameters ${ }^{29}$ in keeping with a negative role of iron levels and metabolism with RBC storage quality and post-transfusion efficacy. ${ }^{30}$ Moreover, $\beta$ Thal $^{+}$RBC are intrinsically different from control, with reduced cellular volume and increased surface:volume ratio. ${ }^{31}$ Altered ion transport homeostasis leading to efflux of osmotically active component $\mathrm{s}^{32}$ underlies to great extent this phenotype. In support, serum levels of osmotically active ions in $\beta$ Thal $^{+}$ exhibit significant correlation with RBC osmotic fragility. ${ }^{33}$ Under normal membrane and skeletal networks (manuscript in preparation) these features contribute to increased resistance to osmotic (and probably to mechanical) stress.

The biological networks suggested that the resistance of stored RBC to osmotic stress is a multifactorial phenotype exhibiting numerous correlations with the baseline RBC physiology (such as the energy metabolism) especially in the case of $\beta \mathrm{Thal}^{+} \mathrm{RBC}$. Indeed, the levels of several biochemical and metabolic features of $\beta T_{\text {Thal }}{ }^{+} \mathrm{RBC}$ in vivo exhibited strong correlations with those of osmotic hemolysis throughout storage, including L-arginin ${ }^{34}$ and sphingosine-1-phosphate, which significantly differed between the two groups under examination at baseline. Of note, similar correlations were also detected in G6PDdefficient donors. ${ }^{35}$ This observation suggests a genetically determined aspect of osmotic hemolysis phenotype in stored RBC that deserves further examination. Since osmotic fragility is strongly donor-specific (storage levels are proportional to baseline levels in vivo), ${ }^{17}$ the superior osmotic hemolysis (and consequently part of storage hemolysis) of $\beta T_{h a l^{+}}$RBC was rather anticipated. Resistance to oxidative hemolysis at late storage seemed to follow the fluctuation in ROS levels at the same period, as well as the modulation of purine and arginine metabolism, previously reported in control donor groups. ${ }^{36}$ Osmotic stress and additional hemolysis triggers may be further associated with the specific protein composition of RBC membrane in $\beta \mathrm{Thal}^{+}$, since our preliminary analysis revealed variations in proteins critically involved in cell volume regulation and transmembrane water/cation flows responsive to osmotic and mechanical stimuli, including aquaporin and piezo. Variation in some of these transporters has already been reported in thalassaemic or other anemic subjects. ${ }^{37}$ Our study (Online Supplementary Figure S1A) showed for the first time that resistance to both spontaneous and induced hemolysis is a distinctive feature of $\beta \mathrm{Thal}^{+} \mathrm{RBC}$ storability. However, diversity in $\beta$ Thal $^{+}$mutations worldwide should be also taken into consideration. Indeed, certain $\beta \mathrm{Thal}^{+}$donors exhibited control baseline levels of RBC osmotic fragility (Table 1). The effects of such genetic variation on $\beta$ Thal $^{+} \mathrm{RBC}$ storability and performance deserve further evaluation by future studies in various ethnic groups.

Apart from hemolysis, PS exposure (a potent signal for RBC clearance in vivo) was also lower in $\beta T_{h a l^{+}} \mathrm{RBC}$ at late storage, when ROS accumulation and membrane expression of phospholipid scramblase ${ }^{38}$ were also low. In contrast, $\mathrm{Ca}^{2+}$ concentration was higher in $\beta \mathrm{Thal}^{+} \mathrm{RBC}$ at baseline (and slightly higher during storage) compared to controls, consistent with the distorted divalent cation homeostasis reported in $\beta$ Thal $^{+}{ }^{+39}$ This finding deserves further attention in the light of $\beta \mathrm{Thal}^{+}$-specific variation in $\mathrm{Ca}^{2+}$-dependent membrane proteins that are involved in critical signaling events in RBC. Finally, alterations in the hexosamine pathway in $\beta \mathrm{Thal}^{+} \mathrm{RBC}$ are suggestive of either up-regulation of UDP-N-acetyl-glucosamine usage for O-GlcNAcylation or decreased synthesis through blockade of the late steps in this biosynthetic pathway. To the best of our knowledge, this is the first report of this pathway being affected in $\beta \mathrm{Thal}^{+}$. Interestingly, $\mathrm{O}$ GlcNAcylation is a common post-translational modification in Plasmodium falciparum proteins following malaria infection, to the extent that therapeutic blockade of this pathway has been proposed as an intervention to shorten the life cycle of the Plasmodium. ${ }^{40}$ As such, our observa- 
tions may contribute to explain one of the molecular mechanisms through which $\beta \mathrm{Thal}^{+}$protects from malaria infection. ${ }^{22}$

Reports on the oxidative profile of $\beta \mathrm{Thal}^{+} \mathrm{RBC}$ and on the plasma antioxidant capacity in vivo are quite contradicting. ${ }^{12}$ Many studies, however, pointed to increased activity and/or upregulation of several antioxidant enzymes and proteins ${ }^{41}$ in $\beta$ Thal $^{+} \mathrm{RBC}$ as a possible adaptation to mild sustained oxidative stress. Metabolism exhibits a similar adaptive response according to the currently reported data showing lower baseline levels of oxidant stress-induced purine deamination, higher levels of sulfur-containing metabolites (methionine and cysteine), increase in glycolysis upstream of pyruvate kinase and an apparent increase in fluxes through the PPP in $\beta T_{h a l^{+}}$ $\mathrm{RBC}$. Combined with increases in PPP-derived reducing equivalents (NADPH), in the absence of significant alterations of total glutathione pools and ratios of oxidized/reduced forms, these data are suggestive of an overall higher antioxidant armamentarium in $\beta \mathrm{Thal}^{+}$ RBC. "Training" to mild oxidative stress in vivo may modulate $\beta$ Thal $^{+}$RBC storability ${ }^{41}$ as evidenced by ROS accumulation, oxidative hemolysis, and oxidative defects to membrane components. In the same context, the membrane profile of stress markers and the low intracellular allantoin $^{42}$ are suggestive of the protective action of molecular chaperones and other antioxidant molecules, such as urate, present in excess in $\beta T_{h a l^{+}} \mathrm{RBC}$. Despite the fact that urate can act as a pro- or anti-oxidant factor, its minimal oxidation to allantoin at the same period of low ROS generation in $\beta \mathrm{Thal}^{+} \mathrm{RBC}$ points towards its antioxidant effect during storage. Moreover, it mostly accounted for the elevated antioxidant capacity of $\beta \mathrm{Thal}^{+}$ plasma/supernatant. Network paired analysis of fresh versus stored samples confirmed that the extracellular antioxidant capacity (like osmotic fragility) is an almost "heritable" feature of RBC units in both donor groups, ${ }^{43}$ and further "signs" $\beta \mathrm{Thal}^{+}$status in stored RBC (Online Supplementary Figure S1). Of note, the levels of TAC and UAdAC in $\beta \mathrm{Thal}^{+}$units exhibited inverse correlations with acyl-carnitine RBC metabolites, such as the redox related acetyl-carnitine. These metabolites have been suggested as markers of osmotic fragility in blood donors undergoing testosterone-replacement therapy, ${ }^{44}$ consistent with a role of this pathway in the Lands cycle-dependent remodeling of oxidized membrane lipids.

Genetic variation among donors may have a positive or negative impact on transfusion performance. G6PD-deficient $\mathrm{RBC}$, for instance, are susceptible to hemolysis and oxidative stress post-transfusion, ${ }^{6}$ and exhibit lower 24hour recovery in autologous transfusion recipients. Similarly, sickle cell trait RBC were shown to have reduced recovery in animal models. ${ }^{28}$ Quite to the contrary, the unique storability of $\beta \mathrm{Thal}^{+} \mathrm{RBC}$ is suggestive of good post-transfusion recovery. Apart from the low intracellular levels of hypoxanthine, a metabolic marker of storage lesion exhibiting negative correlation with recovery in vivo, ${ }^{45}$ the low extracellular $\mathrm{Hb}$ /potassium and $\mathrm{RBC}$ removal signaling are in favor of therapeutic outcomes mainly through preservation of nitric oxide bioavailability and alleviation of hemolysis/iron-associated toxicity, including inflammatory responses and bacterial infections. ${ }^{46}$ In addition, the advantageous redox phenotype of stored $\beta \mathrm{Thal}^{+}$is expected to promote their functionality in vivo and to ameliorate oxidative stress- associated adverse clinical outcomes in susceptible patients with G6PD-deficiency and sickle cell disease. ${ }^{47}$ Finally, the modified arginine/nitric oxide metabolism (involved in the induction of $\mathrm{HbF}^{48}$ ) in $\beta \mathrm{Thal}^{+} \mathrm{RBC}$ during storage may be relevant to their transfusion in susceptible recipients, including hemoglobinopathies, cancer, lung and cardiovascular disease patients. In the light of those results, we have initiated a study on post-transfusion performance of $\beta$ Thal ${ }^{+} \mathrm{RBC}$.

Heterozygotes for $\beta \mathrm{Thal}^{+}$constitute a non-negligible proportion of blood donors not only in the Mediterranean, but also in Africa, Asia and in under-represented minorities of US. ${ }^{49}$ Their typical profile of low $\mathrm{Hb}$ concentration, and thus, of lower dose of $\mathrm{Hb}$ per unit of $\mathrm{RBC}$, would predispose to reduced $\mathrm{Hb}$ increment and lower efficacy of the transfusion therapy compared to control. However, the currently presented data show that $\beta \mathrm{Thal}^{+} \mathrm{RBC}$ are unique in terms of susceptibility to hemolysis, redox homeostasis and nitrogen/hexosaminerelated metabolism during storage. This favorable storability and thereof improved quality of stored $\beta T h a l^{+} \mathrm{RBC}$ may counterpoise the negative effects of lower $\mathrm{Hb}$ dose on the recipient. Despite the fact that further studies are needed to clarify the post-transfusion performance of $\beta \mathrm{Thal}^{+} \mathrm{RBC}$ in the context of intra-group genetic heterogeneity and recipient variation, the currently reported data may allow considering $\beta \mathrm{Thal}^{+}$individuals as a novel, high quality, "good storer" group. Indeed, $\beta$ Thal ${ }^{+}$RBC may be safely stored for long time periods before transfusion, highlighting the notion of molecular versus storage age of blood. ${ }^{5}$ Moreover, $\beta \mathrm{Thal}^{+} \mathrm{RBC}$ are probably ideal candidates for alternative storage strategies, like cryopreservation, which involves a deglycerolization step during which RBC are highly susceptible to hypo-osmotic lysis. ${ }^{50}$ These promising data and potentials highlight the emergence of $\beta \mathrm{Thal}^{+}$donors for a distinct and potentially valuable role in blood transfusions.

\section{Disclosures}

Though unrelated to the contents of this manuscript, ADA declares that he is a founder of Omix Technologies Inc and Altis Biosciencens LLC and a consultant for Hemanext Inc. All other authors declare no conflicts of interest.

\section{Contributions}

VLT and ATA are co-first authors; VLT and MHA designed the study; VLT, ATA, MHA and ADA wrote the manuscript; $V L T, A T A$ and $A G K$ performed the physiological and biochemical experiments; $D S, F C, L B$ and $F G$ performed the metabolomics analyses; $M D$ performed the proteomics experiments; MHA and PR performed the flow cytometry analyses; VLT, ATA, MHA and ADA analyzed the data; AV, VS, KS, and $A G K$ provided project support and organized participant recruitment; $M D, O E T, I S P, K S, A G K$ and $A D A$ provided expert knowledge.

\section{Acknowledgements}

The authors would like to thank all blood donors that voluntarily participated in this study; M.S. Jacovides Hellas S.A. for the kind offer of the LTRC blood bags; Dr Pantelis Constantoulakis and Dr Stavros Bournazos (Genotypos Science Labs) for their contribution in the mutation identification; the NKUA students Dimitrios G. Karadimas (MSc) and Christos Christogeorgos for their participation in a part of the physiological experiments performed; Dr Hara Georgatzakou, Mrs 
Maria Sioumala and the UniWA students Efthimia Pavlou, Panagiotis Drosos, Maria Tsoumpeli, Eirini Tsolou and Kariofyllis Karamperis for their contribution in blood sampling and blood cell counting measurements performed in fresh samples or stored RBC units.

\section{Funding}

This project has received funding from the Hellenic Foundation for Research and Innovation (HFRI) and the General Secretariat for Research and Technology (GSRT), under grant agreement No 2032 (VLT).

\section{References}

1. Tzounakas VL, Kriebardis AG, Papassideri IS, Antonelou MH. Donor-variation effect on red blood cell storage lesion: A close relationship emerges. Proteomics Clin Appl. 2016;10(8):791-804.

2. Kanias T, Lanteri MC, Page GP, et al. Ethnicity, sex, and age are determinants of red blood cell storage and stress hemolysis: results of the REDS-III RBC-Omics study. Blood Adv. 2017;1(15):1132-1141.

3. Stefanoni D, Fu X, Reisz JA, et al. Nicotine exposure increases markers of oxidant stress in stored red blood cells from healthy donor volunteers. Transfusion. 2020;60(6):11601174.

4. Roubinian NH, Plimier C, Woo JP, et al. Effect of donor, component, and recipient characteristics on hemoglobin increments following red blood cell transfusion. Blood. 2019;134(13):1003-1013.

5. D'Alessandro A, Zimring JC, Busch M. Chronological storage age and metabolic age of stored red blood cells: are they the same? Transfusion. 2019;59(5):1620-1623.

6. Tzounakas VL, Kriebardis AG, Georgatzakou HT, et al. Glucose 6-phosphate dehydrogenase deficient subjects may be better "storers" than donors of red blood cells. Free Radic Biol Med. 2016;96:152-165.

7. Francis RO, D'Alessandro A, Eisenberger A, et al. Donor glucose-6-phosphate dehydrogenase deficiency decreases blood quality for transfusion. J Clin Invest. 2020;130(5): 2270-2285.

8. Acquaye CT, Oldham JH, Konotey-Ahulu FI. Blood-donor homozygous for hereditary persistence of fetal haemoglobin. Lancet. 1977;1(8015):796-797.

9. Singha K, Taweenan W, Fucharoen G, Fucharoen S. Erythrocyte indices in a large cohort of beta-thalassemia carrier: Implication for population screening in an area with high prevalence and heterogeneity of thalassemia. Int J Lab Hematol. 2019;41 (4):513-518.

10. Hillier J, Hoffman JF, Parpart AK, Wolman IJ. Ultrastructure of erythrocyte membranes in thalassemia major and minor. Blood. 1956;11(10):946-956.

11. Ficarra S, Tellone E, Giardina B, et al. Derangement of erythrocytic AE1 in betathalassemia by caspase 3: pathogenic mechanisms and implications in red blood cell senescence. J Membr Biol. 2009;228(1):4349.

12. Manafikhi H, Drummen G, Palmery M, Peluso I. Total antioxidant capacity in betathalassemia: a systematic review and metaanalysis of case-control studies. Crit Rev Oncol Hematol. 2017;110:35-42.

13. Fortier N, Snyder LM, Garver F, Kiefer C, McKenney J, Mohandas N. The relationship between in vivo generated hemoglobin skeletal protein complex and increased red cell membrane rigidity. Blood. 1988;71(5): 1427-1431.

14. Pantaleo A, De Franceschi L, Ferru E, Vono R, Turrini F. Current knowledge about the functional roles of phosphorylative changes of membrane proteins in normal and diseased red cells. J Proteomics. 2010;73(3):445455.

15. Mandal D, Baudin-Creuza V, Bhattacharyya A, et al. Caspase 3-mediated proteolysis of the $\mathrm{N}$-terminal cytoplasmic domain of the human erythroid anion exchanger 1 (band 3). J Biol Chem. 2003;278(52):52551-52558.

16. Schrier SL, Rachmilewitz E, Mohandas N. Cellular and membrane properties of alpha and beta thalassemic erythrocytes are different: implication for differences in clinical manifestations. Blood. 1989;74(6):21942202.

17. Tzounakas VL, Anastasiadi AT, Karadimas DG, et al. Temperature-dependent haemolytic propensity of CPDA-1 stored red blood cells vs whole blood - Red cell fragility as donor signature on blood units. Blood Transfus. 2017;15(5):447-455.

18. Tzounakas VL, Georgatzakou HT, Kriebardis AG, et al. Donor variation effect on red blood cell storage lesion: a multivariable, yet consistent, story. Transfusion. 2016;56(6):1274-1286.

19. Falco C, Vaya A, Iborra J, Moreno I, Palanca S, Aznar J. Erythrocyte aggregability and disaggregability in thalassemia trait carriers analyzed by a laser backscattering technique. Clin Hemorheol Microcirc 2003;28(4):245-249.

20. Triantafyllou AI, Vyssoulis GP, Karpanou EA, et al. Impact of beta-thalassemia trait carrier state on cardiovascular risk factors and metabolic profile in patients with newly diagnosed hypertension. J Hum Hypertens. 2014;28(5):328-332

21. Maioli M, Pettinato S, Cherchi GM, et al. Plasma lipids in beta-thalassemia minor Atherosclerosis. 1989;75(2-3):245-248.

22. Kuesap J, Chaijaroenkul W, Rungsihirunrat K, Pongjantharasatien K, Na-Bangchang K. Coexistence of malaria and thalassemia in malaria endemic areas of Thailand. Korean J Parasitol. 2015;53(3):265-270.

23. Harboe M. A method for determination of hemoglobin in plasma by near-ultraviolet spectrophotometry. Scand J Clin Lab Invest. 1959:11:66-70.

24. Reisz JA, Chessler KM, Dzieciatkowska M, D'Alessandro A, Hansen KC. Blood and plasma proteomics: targeted quantitation and posttranslational redox modifications. Methods Mol Biol. 2017;1619:353-371.

25. Nemkov T, Reisz JA, Gehrke S, Hansen KC, D'Alessandro A. High-throughput metabolomics: isocratic and gradient mass spectrometry-based methods. Methods Mol Biol. 2019;1978:13-26.

26. Nemkov T, Hansen KC, D'Alessandro A. A three-minute method for high-throughput quantitative metabolomics and quantitative tracing experiments of central carbon and nitrogen pathways. Rapid Commun Mass Spectrom. 2017;31(8):663-673.

27. Sun K, Zhang Y, D'Alessandro A, et al. Sphingosine-1-phosphate promotes erythrocyte glycolysis and oxygen release for adaptation to high-altitude hypoxia. Nat Commun. 2016;7:12086
28. Osei-Hwedieh DO, Kanias T, Croix CS, et al. Sickle cell trait increases red blood cell storage hemolysis and post-transfusion clearance in mice. EBioMedicine. 2016;11:239-248

29. Kanias T, Stone M, Page GP, et al. Frequent blood donations alter susceptibility of red blood cells to storage- and stress-induced hemolysis. Transfusion. 2019;59(1):67-78.

30. Howie HL, Hay AM, de Wolski K, et al Differences in Steap3 expression are a mechanism of genetic variation of $\mathrm{RBC}$ storage and oxidative damage in mice. Blood Adv. 2019;3(15):2272-2285

31. Gunn RB, Silvers DN, Rosse WF. Potassium permeability in -thalassemia minor red blood cells. J Clin Invest. 1972;51(5):10431050

32. Olivieri O, De Franceschi L, Capellini MD, Girelli D, Corrocher R, Brugnara C. Oxidative damage and erythrocyte membrane transport abnormalities in thalassemias. Blood. 1994:84(1):315-320.

33. Tzounakas V, Drossos P, Karadimas D, et al. Hematological and physiological characteristics of regular blood donors with beta-thalassemia traits. Vox Sang. 2019;114(s1): abstract P-059.

34. Belfiore F, Meldolesi J, Calcara G. Erythrocyte enzymes in thalassaemia and thalassodrepanocytosis. Acta Haematol 1965;34(6):329-337.

35. Reisz JA, Tzounakas VL, Nemkov T, et al Metabolic linkage and correlations to storage capacity in erythrocytes from glucose 6 phosphate dehydrogenase-deficient donors. Front Med (Lausanne). 2017;4:248.

36. D'Alessandro A, Fu X, Kanias $T$, et al Donor sex, age and ethnicity impact stored red blood cell antioxidant metabolism through mechanisms in part explained by glucose 6-phosphate dehydrogenase levels and activity. Haematologica. 2021;106(5) 1290-1302.

37. Omar AK, Ahmed KA, Helmi NM, et al. The sensitivity of $\mathrm{Na}(+), \mathrm{K}(+)$ ATPase as an indicator of blood diseases. Afr Health Sci. 2017;17(1):262-269.

38. Wesseling MC, Wagner-Britz L, Nguyen DB, et al. Novel insights in the regulation of phosphatidylserine exposure in human red blood cells. Cell Physiol Biochem. 2016;39 (5):1941-1954

39. Shalev O, Mogilner S, Shinar E, Rachmilewitz EA, Schrier SL. Impaired erythrocyte calcium homeostasis in beta-thalassemia. Blood. 1984;64(2):564-566.

40. Kupferschmid M, Aquino-Gil MO, ShamsEldin $\mathrm{H}$, et al. Identification of $\mathrm{O}$ GlcNAcylated proteins in Plasmodium falciparum. Malar J. 2017;16(1):485.

41. Teran MM, Monaco ME, Lazarte SS, et al Genetic regulation of redox balance in betathalassemia Trait. Hemoglobin. 2020;44(2): 122-127.

42. Kand'ar R, Zakova P. Allantoin as a marker of oxidative stress in human erythrocytes. Clin Chem Lab Med. 2008;46(9):12701274

43. Tzounakas VL, Karadimas DG, Anastasiadi AT, et al. Donor-specific individuality of red 
blood cell performance during storage is partly a function of serum uric acid levels. Transfusion. 2018;58(1):34-40.

44. Alexander K, Hazegh K, Fang F, et al. Testosterone replacement therapy in blood donors modulates erythrocyte metabolism and susceptibility to hemolysis in cold storage. Transfusion. 2021;61(1):108-123.

45. Nemkov T, Sun K, Reisz JA, et al. Hypoxia modulates the purine salvage pathway and decreases red blood cell and supernatant levels of hypoxanthine during refrigerated stor- age. Haematologica. 2018;103(2):361-372.

46. Hod EA, Zhang N, Sokol SA, et al Transfusion of red blood cells after prolonged storage produces harmful effects that are mediated by iron and inflammation. Blood. 2010;115(21):4284-4292.

47. Kanias T, Acker JP. Biopreservation of red blood cells--the struggle with hemoglobin oxidation. FEBS J. 2010;277(2):343-356.

48. Cokic VP, Smith RD, Beleslin-Cokic BB, et al. Hydroxyurea induces fetal hemoglobin by the nitric oxide-dependent activation of soluble guanylyl cyclase. J Clin Invest. 2003;111(2):231-239.

49. Meena LP, Kumar K, Singh VK, Bharti A, Rahman SK, Tripathi K. Study of mutations in beta-thalassemia trait among blood donors in eastern Uttar Pradesh. J Clin Diagn Res. 2013;7(7):1394-1396.

50. Anastasiadi AT, Tzounakas VL, Kriebardis AG, Stamoulis KE, Seghatchian J, Antonelou $\mathrm{MH}$. When I need you most: frozen red blood cells for transfusion. Transfus Apher Sci. 2020;59(3):102786. 